\title{
Estimation of Parameters of Boundary Value Problems for Linear Ordinary Differential Equations with Uncertain Data
}

\author{
Yury Shestopalov ${ }^{*}$, Yury Podlipenko², Olexandr Nakonechnyi² \\ ${ }^{1}$ Gävle University, Gävle, Sweden \\ ${ }^{2}$ Kyiv National University, Kyiv, Ukraine \\ Email: ‘youri.shestopalov@kau.se, yourip@mail.ru, nakonechniy@unicyb.kiev.ua
}

Received 13 February 2014; revised 13 March 2014; accepted 18 March 2014

Copyright @ 2014 by authors and Scientific Research Publishing Inc.

This work is licensed under the Creative Commons Attribution International License (CC BY). http://creativecommons.org/licenses/by/4.0/

(c) (i) Open Access

\section{Abstract}

In this paper we construct optimal, in certain sense, estimates of values of linear functionals on solutions to two-point boundary value problems (BVPs) for systems of linear first-order ordinary differential equations from observations which are linear transformations of the same solutions perturbed by additive random noises. It is assumed here that right-hand sides of equations and boundary data as well as statistical characteristics of random noises in observations are not known and belong to certain given sets in corresponding functional spaces. This leads to the necessity of introducing minimax statement of an estimation problem when optimal estimates are defined as linear, with respect to observations, estimates for which the maximum of mean square error of estimation taken over the above-mentioned sets attains minimal value. Such estimates are called minimax mean square or guaranteed estimates. We establish that the minimax mean square estimates are expressed via solutions of some systems of differential equations of special type and determine estimation errors.

\section{Keywords}

Optimal Minimax Mean Square Estimates, Uncertain Data, Two-Point Boundary Value Problems, Random Noises, Observations

\section{Introduction}

The theory of finding estimates of solutions to stochastic differential equations has been intensively developing

\footnotetext{
"Corresponding author.
}

How to cite this paper: Shestopalov, Y., et al. (2014) Estimation of Parameters of Boundary Value Problems for Linear Ordinary Differential Equations with Uncertain Data. Advances in Pure Mathematics, 4, 118-146. 
since the classical works of Kalman and Bucy [1] [2]. This theory found numerous applications in the treatment of the results of experiments in physics, biology, medicine, and many other areas of science and technology. Such successful and broad applications are explained by the fact that Kalman-Bucy methods provide differential equations for optimal mean square estimates which can be solved numerically in the real-time mode. At the same time, it should be noted that Krasovskii and Kurzhanskii proposed in [3] [4] an alternative approach to estimating the solutions of differential equations where perturbations and inaccuracies of additional data about solution were not known and the only thing given was that they belong to a certain domain.

Let us formulate a general approach to the problem of minimax estimation. If a state of a system is described by a linear ordinary differential equation

$$
\frac{\mathrm{d} x(t)}{\mathrm{d} t}=A x(t)+B v_{1}(t), \quad x\left(t_{0}\right)=x_{0},
$$

and a function $y(t)$ is observed in a time interval $\left[t_{0}, T\right]$, where $y(t)=H x(t)+v_{2}(t), \quad x(t) \in \mathbb{R}^{n}$, $v_{2} \in \mathbb{R}^{m}, \quad y \in \mathbb{R}^{m}$, and $A, B, H$ are known matrices, the minimax estimation problem consists in the most accurate determination of a function $x(t)$ at the "worst" realization of unknown quantities $\left(x_{0}, v_{1}(\cdot), v_{2}(\cdot)\right)$ taken from a certain set. N.N. Krasovskii was the first who stated this problem in [3]. Under different constraints imposed on function $v_{2}(t)$ and for known function $v_{1}(t)$ he proposed various methods of estimating inner products $(a, x(T))$, where $a \in \mathbb{R}^{n}$, in the class of operations linear with respect to observations that minimize the maximal error. Later these estimates were called minimax a priori or guaranteed estimates (see [3] [4]).

This theory was further developed in the works of Chernous'ko, Pshenichnyi, Kuntzevich, Nakonechnyi, Kirichenko, Podilipenko, and their disciples; one may refer e.g. to [4]-[10] and the bibliography therein.

We note that the duality principle elaborated in [3] [4], and [5] proved its efficiency for the determination of minimax estimates [5]. According to this principle, finding minimax a priori estimates can be reduced to a certain problem of optimal control of a system; this approach enabled one to obtain, under certain restrictions, recurrent equations, namely, the minimax Kalman-Bucy filter (see [5]).

The essential results within the frames of $H_{\infty}$-theory are obtained in [11].

In this paper, we study estimation of solutions of boundary value problems (BVPs) for ordinary differential equations at fixed points of interval from additional data about their solutions. Such settings may be considered as inverse problems when additional data are given with errors. We assume that these errors are random with unknown correlation functions. Similar problems arise in data processing of observations of the objects or processes described by BVPs for ordinary differential equations with unknown perturbations of the right-hand sides or boundary conditions. We solve the estimation problems using guaranteed linear estimates that minimize maximal mean square estimation errors. It is shown that optimal guaranteed estimates are expressed via solutions to special BVPs for ordinary differential equations.

\section{Preliminaries and Auxiliary Results}

Assume that it is given a vector-function $f(t)=\left(f_{1}(t), f_{2}(t), \cdots, f_{n}(t)\right)^{\mathrm{T}}$ with the components belonging to space $L^{2}(0, T)$ and vectors $f_{0}=\left(f_{1}^{(0)}, f_{2}^{(0)}, \cdots, f_{m}^{(0)}\right)^{\mathrm{T}} \in \mathbb{R}^{m}$ and $f_{1}=\left(f_{1}^{(1)}, f_{2}^{(1)}, \cdots, f_{n-m}^{(1)}\right)^{\mathrm{T}} \in \mathbb{R}^{n-m}$. Consider the following BVP: find a vector-function $\varphi(t)=\left(\varphi_{1}(t), \varphi_{2}(t), \cdots, \varphi_{n}(t)\right)^{\mathrm{T}} \in\left(H^{1}(0, T)\right)^{n}$ that satisfies a system of linear first-order ordinary differential equations

$$
L \varphi(t) \equiv \frac{\mathrm{d} \varphi}{\mathrm{d} t}+A \varphi=f(t), t \in(0, T), L=\frac{\mathrm{d}}{\mathrm{d} t}+A,
$$

almost everywhere on an interval $(0, T)$ and the boundary conditions

$$
B_{0} \varphi(0)=f_{0}, \quad B_{1} \varphi(T)=f_{1}
$$

at the points 0 and $T$. Here $A=A(t)$ is an $n \times n$ matrix with the entries $a_{i j}=a_{i j}(t)$ continuous on $[0, T]$; 
$\frac{\mathrm{d} \varphi(t)}{\mathrm{d} t}=\left(\frac{\mathrm{d} \varphi_{1}(t)}{\mathrm{d} t}, \frac{\mathrm{d} \varphi_{2}(t)}{\mathrm{d} t}, \cdots, \frac{\mathrm{d} \varphi_{n}(t)}{\mathrm{d} t}\right)^{\mathrm{T}} ; \quad B_{0}=\left\{b_{r s}^{(0)}\right\}, \quad r=\overline{1, m}, \quad s=\overline{1, n}, \quad$ and $\quad B_{1}=\left\{b_{r s}^{(1)}\right\}, \quad r=\overline{1, n-m}$, $s=\overline{1, n}$, are $m \times n$ and $(n-m) \times n$ matrices of rank $m$ and $n-m$, respectively; upper index $\mathrm{T}$ denotes transposition of a matrix or a vector and the upper bar throughout the whole text of the paper that e.g. index $r$ takes all values from 1 to $n-m ; H^{1}(a, b)$ is a space of functions absolutely continuous on $[a, b]$ for which the derivative that exists almost everywhere on $(a, b)$ belongs to space $L^{2}(a, b)$; and $\left(H^{1}(0, T)\right)^{n}=\frac{n \text { times }}{H^{1}(0, T) \times \cdots \times H^{1}(0, T)}$.

The problem of finding a function $\varphi(t)$ that satisfies on $(0, T)$ the equation

$$
\frac{\mathrm{d} \varphi(t)}{\mathrm{d} t}+A \varphi(t)=0
$$

and the boundary conditions

$$
B_{0} \varphi(0)=0, \quad B_{1} \varphi(T)=0
$$

will be called the homogeneous BVP corresponding to BVP (0.1), (0.2).

The solution $\varphi(t) \equiv 0$ to homogeneous BVP (0.3), (0.4) is called the trivial solution.

BVP (0.1), (0.2) can be written in a scalar form:

$$
\begin{gathered}
\varphi_{1}^{\prime}(t)+a_{11} \varphi_{1}(t)+a_{12} \varphi_{2}(t)+\cdots+a_{1 n} \varphi_{n}(t)=f_{1}(t), \\
\varphi_{2}^{\prime}(t)+a_{21} \varphi_{1}(t)+a_{22} \varphi_{2}(t)+\cdots+a_{2 n} \varphi_{n}(t)=f_{1}(t), \\
\vdots \\
\varphi_{n}^{\prime}(t)+a_{n 1} \varphi_{1}(t)+a_{n 2} \varphi_{2}(t)+\cdots+a_{n n} \varphi_{n}(t)=f_{n}(t), \\
U_{i}(\varphi):=\sum_{q=1}^{n} b_{i q}^{(0)} \varphi_{q}(0)=f_{i}^{(0)}, i=\overline{1, m}, \\
U_{m+i}(\varphi):=\sum_{q=1}^{n} b_{i q}^{(1)} \varphi_{q}(T)=f_{i}^{(1)}, \quad i=\overline{1, n-m} .
\end{gathered}
$$

Let

$$
\varphi^{(i)}(t)=\left(\varphi_{1}^{(i)}(t), \varphi_{2}^{(i)}(t), \cdots, \varphi_{n}^{(i)}(t)\right)^{\mathrm{T}}, \quad i=\overline{1, n},
$$

be a fundamental system of solutions to (0.3) (for the definition, see e.g. [12] p. 179). Then the solutions to (0.3), (0.4) have the form

$$
\varphi(t)=c_{1} \varphi^{(1)}(t)+c_{2} \varphi^{(2)}(t)+\cdots+c_{n} \varphi^{(n)}(t),
$$

where, by virtue of (0.4), constants $c_{1}, c_{2}, \cdots, c_{n}$ must be such that

$$
\begin{gathered}
c_{1} U_{1}\left(\varphi^{(1)}\right)+c_{2} U_{1}\left(\varphi^{(2)}\right)+\cdots+c_{n} U_{1}\left(\varphi^{(n)}\right)=0, \\
c_{1} U_{2}\left(\varphi^{(1)}\right)+c_{2} U_{2}\left(\varphi^{(2)}\right)+\cdots+c_{n} U_{2}\left(\varphi^{(n)}\right)=0, \\
\vdots \\
c_{1} U_{n}\left(\varphi^{(1)}\right)+c_{2} U_{n}\left(\varphi^{(2)}\right)+\cdots+c_{n} U_{n}\left(\varphi^{(n)}\right)=0 .
\end{gathered}
$$

Thus, if the matrix 


$$
\left(\begin{array}{cccc}
U_{1}\left(\varphi^{(1)}\right) & U_{1}\left(\varphi^{(2)}\right) & \cdots & U_{1}\left(\varphi^{(n)}\right) \\
U_{2}\left(\varphi^{(1)}\right) & U_{2}\left(\varphi^{(2)}\right) & \cdots & U_{2}\left(\varphi^{(n)}\right) \\
\vdots & \vdots & \ddots & \cdots \\
U_{n}\left(\varphi^{(1)}\right) & U_{n}\left(\varphi^{(2)}\right) & \cdots & U_{n}\left(\varphi^{(n)}\right)
\end{array}\right)
$$

has rank $n$, the homogeneous BVP has only the trivial solution. The inverse statement is also valid: if the homogeneous BVP has only the trivial solution then the rank of matrix (0.9) equals $n$ (see, for example [13]).

Assume in what follows that homogeneous BVP (0.3), (0.4) corresponding to BVP (0.1), (0.2) has only the trivial solution or what is the same that matrix (0.9) has rank $n$. As is known [13], under this assumption, initial BVP (0.1), (0.2) is uniquely solvable at any right-hand sides $f(t)=\left(f_{1}(t), f_{2}(t), \cdots, f_{n}(t)\right)^{\mathrm{T}}$, $f_{0}=\left(f_{1}^{(0)}, f_{2}^{(0)}, \cdots, f_{m}^{(0)}\right)^{\mathrm{T}} \in \mathbb{R}^{m}$, and $f_{1}=\left(f_{1}^{(1)}, f_{2}^{(1)}, \cdots, f_{n-m}^{(1)}\right)^{\mathrm{T}} \in \mathbb{R}^{n-m}$.

Formulate the notion of a BVP conjugate to $(0.1),(0.2)$. To this end, introduce the following designations: $E_{k}$ is the $k \times k$ unit matrix; $O_{k, r}$ is the $k \times r$ null matrix; $B_{01}=\left\{b_{r i_{k}}^{(0)}\right\}, \quad r=\overline{1, m}, k=\overline{1, m}$, is a square nondegenerate $m \times m$ submatrix of the matrix $B_{0}=\left\{b_{r s}^{(0)}\right\}, \quad r=\overline{1, m}, \quad s=\overline{1, n} ; \quad B_{02}=\left\{b_{r j_{l}}^{(0)}\right\}, \quad r=\overline{1, m}$, $l=\overline{1, n-m}$, is an $m \times(n-m)$ submatrix of $B_{0}$ obtained as a result of deleting in $B_{0}$ all columns of matrix $B_{01}$ (so that $\left.\left\{j_{1}, \cdots, j_{n-m}\right\}=\{1, \cdots, n\} \backslash\left\{i_{1}, \cdots, i_{m}\right\}\right) ; \hat{B}_{0}=\left(-B_{02}^{\mathrm{T}}\left(B_{01}^{\mathrm{T}}\right)^{-1}, E_{n-m}\right)$ is an $(n-m) \times n$ matrix such that its $i_{k}$ th column equals $k$ th column of matrix $-B_{02}^{\mathrm{T}}\left(B_{01}^{\mathrm{T}}\right)^{-1}$ (its size is $(n-m) \times n$ ), $k=\overline{1, m}$, and $j_{l}$ th column equals $l$ th column of matrix $E_{n-m}, \quad l=\overline{1, n-m} ; \quad \bar{B}_{0}=\left(\left(B_{01}^{\mathrm{T}}\right)^{-1}, O_{m, n-m}\right)$ is an $m \times n$ matrix such that its $i_{k}$ th column equals $k$-th column of matrix $\left(B_{01}^{\mathrm{T}}\right)^{-1}, k=\overline{1, m}$, and $j_{l}$ th column equals $l$ th column of matrix $O_{m, n-m}, \quad l=\overline{1, n-m} ; \quad \tilde{B}_{0}=\left(O_{n-m, m}, E_{n-m}\right)$ is an $(n-m) \times n$ matrix such that its $i_{k}$ th column equals $k$ th column of matrix $O_{n-m, m}, \quad k=\overline{1, m}$, and $j_{l}$ th column equals $l$ th column of matrix $E_{n-m}, \quad l=\overline{1, n-m}$.

Introduce more similar notations: $\quad B_{11}=\left\{b_{r i_{k}^{\prime}}^{(1)}\right\}, \quad r=\overline{1, n-m}, \quad k=\overline{1, n-m}$, is a square nondegenerate $(n-m) \times(n-m) \quad$ submatrix of the matrix $B_{1}=\left\{b_{r s}^{(1)}\right\}, \quad r=\overline{1, n-m}, \quad s=\overline{1, n} ; \quad B_{12}=\left\{b_{r j i}^{(1)}\right\}, \quad r=\overline{1, n-m}$, $l=\overline{1, m}$, is a $(n-m) \times m$ submatrix of the matrix $B_{1}$ obtained as a result of deleting in $B_{1}$ all columns of matrix $B_{11}$ (so that $\left.\left\{j_{1}^{\prime}, \cdots, j_{m}^{\prime}\right\}=\{1, \cdots, n\} \backslash\left\{i_{1}^{\prime}, \cdots, i_{n-m}^{\prime}\right\}\right) ; \hat{B}_{1}=\left(-B_{12}^{\mathrm{T}}\left(B_{11}^{\mathrm{T}}\right)^{-1}, E_{m}\right)$ is an $m \times n$ matrix such that its $i_{k}^{\prime}$ th column equals $k$ th column of matrix $-B_{12}^{\mathrm{T}}\left(B_{11}^{\mathrm{T}}\right)^{-1}$ (the size of the latter is $\left.m \times(n-m)\right)$, $k=\overline{1, n-m}$, and $j_{l}^{\prime}$ th column equals $l$ th column of matrix $E_{m}, \quad l=\overline{1, m} ; \quad \bar{B}_{1}=\left(\left(B_{11}^{\mathrm{T}}\right)^{-1}, O_{n-m, m}\right)$ is an $(n-m) \times n$ matrix such that its $i_{k}^{\prime}$ th column equals $k$ th column of matrix $\left(B_{11}^{\mathrm{T}}\right)^{-1}, k=\overline{1, n-m}$, and $j_{l}^{\prime}$ th column equals $l$ th column of matrix $O_{n-m, m}, \quad l=\overline{1, m} ; \quad \tilde{B}_{1}=\left(O_{m, n-m}, E_{m}\right)$ is an $m \times n$ matrix such that its $i_{k}^{\prime}$ th column equals $k$ th column of matrix $O_{m, n-m}, k=\overline{1, n-m}$, and $j_{l}^{\prime}$ th column equals lth column of matrix $E_{m}, \quad l=\overline{1, m}$.

By

$$
(u, v)_{N}=\sum_{i=1}^{N} u_{i} v_{i}
$$


we will denote the inner product of vectors $u=\left(u_{1}, \cdots, u_{N}\right)^{\mathrm{T}}$ and $v=\left(v_{1}, \cdots, v_{N}\right)^{\mathrm{T}}$ in the Euclidean space $\mathbb{R}^{N}$.

Then, if $\psi(t)=\left(\psi_{1}(t), \cdots, \psi_{n}(t)\right)^{\mathrm{T}} \in\left(H^{1}(0, T)\right)^{n}$ we have

$$
\begin{aligned}
& \int_{0}^{T}(L \varphi(t), \psi(t))_{n} \mathrm{~d} t=\int_{0}^{T}\left(\frac{\mathrm{d} \varphi(t)}{\mathrm{d} t}+A \varphi(t), \psi(t)\right)_{n} \mathrm{~d} t \\
& =\int_{0}^{T}\left(\frac{\mathrm{d} \varphi(t)}{\mathrm{d} t}, \psi(t)\right)_{n} \mathrm{~d} t+\int_{0}^{T}(A \varphi(t), \psi(t))_{n} \mathrm{~d} t \\
& =\int_{0}^{T} \sum_{i=1}^{n} \frac{\mathrm{d} \varphi_{i}(t)}{\mathrm{d} t} \psi_{i}(t) \mathrm{d} t+\int_{0}^{T} \sum_{i=1}^{n} \sum_{j=1}^{n} a_{i j} \varphi_{j}(t) \psi_{i}(t) \mathrm{d} t \\
& =\left.\sum_{i=1}^{n} \varphi_{i}(t) \psi_{i}(t)\right|_{0} ^{T}-\int_{0}^{T} \sum_{i=1}^{n} \frac{\mathrm{d} \psi_{i}(t)}{\mathrm{d} t} \varphi_{i}(t) \mathrm{d} t+\int_{0}^{T} \sum_{j=1}^{n}\left(\sum_{i=1}^{n} a_{i j} \psi_{i}(t) \varphi_{j}(t)\right) \mathrm{d} t \\
& =(\varphi(T), \psi(T))_{n}-(\varphi(0), \psi(0))_{n}-\int_{0}^{T}\left(\frac{\mathrm{d} \psi(t)}{\mathrm{d} t}, \varphi(t)\right)_{n} \mathrm{~d} t+\int_{0}^{T} \sum_{i=1}^{n}\left(\sum_{j=1}^{n} a_{j i} \psi_{j}(t) \varphi_{i}(t)\right) \mathrm{d} t \\
& =(\varphi(T), \psi(T))_{n}-(\varphi(0), \psi(0))_{n}+\int_{0}^{T}\left(-\frac{\mathrm{d} \psi(t)}{\mathrm{d} t}, \varphi(t)\right)_{n} \mathrm{~d} t+\int_{0}^{T}\left(\varphi(t), A^{\mathrm{T}} \psi(t)\right)_{n} \mathrm{~d} t \\
& =(\varphi(T), \psi(T))_{n}-(\varphi(0), \psi(0))_{n}+\int_{0}^{T}\left(\varphi(t), L^{*} \psi(t)\right)_{n},
\end{aligned}
$$

where the differential operator

$$
L^{*}=-\frac{\mathrm{d}}{\mathrm{d} t}+A^{\mathrm{T}}
$$

will be called formally conjugate to operator $L$.

Let us show that the term $(\varphi(T), \psi(T))_{n}-(\varphi(0), \psi(0))_{n}$ in (0.10) can be represented as

$$
\begin{aligned}
(\psi(T), \varphi(T))_{n}-(\psi(0), \varphi(0))_{n}= & \left(\bar{B}_{1} \psi(T), B_{1} \varphi(T)\right)_{n-m}+\left(\hat{B}_{1} \psi(T), \tilde{B}_{1} \varphi(T)\right)_{m} \\
& -\left(\bar{B}_{0} \psi(0), B_{0} \varphi(0)\right)_{m}-\left(\hat{B}_{0} \psi(0), \tilde{B}_{0} \varphi(0)\right)_{n-m} .
\end{aligned}
$$

Note first that

$$
B_{0} \varphi(0)=\left(\begin{array}{c}
\sum_{q=1}^{n} b_{1 q}^{(0)} \varphi_{q}(0) \\
\vdots \\
\sum_{q=1}^{n} b_{n q}^{(0)} \varphi_{q}(0)
\end{array}\right)=\left(\begin{array}{c}
\sum_{k=1}^{n} b_{1 i_{k}}^{(0)} \varphi_{i_{k}}(0)+\sum_{l=1}^{n-m} b_{1 j_{l}}^{(0)} \varphi_{j_{l}}(0) \\
\vdots \\
\sum_{k=1}^{n} b_{m_{k}}^{(0)} \varphi_{i_{k}}(0)+\sum_{l=1}^{n-m} b_{m j_{l}}^{(0)} \varphi_{j_{l}}(0)
\end{array}\right)=B_{01} \varphi_{1}^{(0)}(0)+B_{02} \varphi_{2}^{(0)}(0),
$$

where

$$
\varphi_{1}^{(0)}(0):=\left(\begin{array}{c}
\varphi_{i_{1}}(0) \\
\vdots \\
\varphi_{i_{m}}(0)
\end{array}\right), \quad \varphi_{2}^{(0)}(0):=\left(\begin{array}{c}
\varphi_{j_{1}}(0) \\
\vdots \\
\varphi_{j_{n-m}}(0)
\end{array}\right) .
$$

Then $\varphi_{1}^{(0)}(0)=B_{01}^{-1} B_{0} \varphi(0)-B_{01}^{-1} B_{02} \varphi_{2}^{(0)}(0)$, and

$$
\begin{aligned}
& (\psi(0), \varphi(0))_{n}=\left(\psi_{1}^{(0)}(0), \varphi_{1}^{(0)}(0)\right)_{m}+\left(\psi_{2}^{(0)}(0), \varphi_{2}^{(0)}(0)\right)_{n-m} \\
& =\left(\psi_{1}^{(0)}(0), B_{01}^{-1} B_{0} \varphi(0)\right)_{m}-\left(\psi_{1}^{(0)}(0), B_{01}^{-1} B_{02} \varphi_{2}^{(0)}(0)\right)_{m}+\left(\psi_{2}^{(0)}(0), \varphi_{2}^{(0)}(0)\right)_{n-m} \\
& =\left(\bar{B}_{0} \psi(0), B_{0} \varphi(0)\right)_{m}-\left(B_{02}^{\mathrm{T}}\left(B_{01}^{\mathrm{T}}\right)^{-1} \psi_{1}^{(0)}(0), \varphi_{2}^{(0)}(0)\right)_{n-m}+\left(\psi_{2}^{(0)}(0), \varphi_{2}^{(0)}(0)\right)_{n-m} \\
& =\left(\bar{B}_{0} \psi(0), B_{0} \varphi(0)\right)_{m}+\left(\left(-B_{02}^{\mathrm{T}}\left(B_{01}^{\mathrm{T}}\right)^{-1}, O_{n-m, n-m}\right) \psi(0), \varphi_{2}^{(0)}(0)\right)_{n-m}+\left(\left(O_{n-m, n-m}, E_{n-m}\right) \psi(0), \varphi_{2}^{(0)}(0)\right)_{n-m},
\end{aligned}
$$


where $\psi_{1}^{(0)}(0)$ and $\psi_{2}^{(0)}(0)$ are vectors composed of components of vector $\psi(0)$ with the numbers equal to the numbers of components of vectors $\varphi_{1}^{(0)}(0)$ and $\varphi_{2}^{(0)}(0)$, respectively. Taking into account that

$$
\left(-B_{02}^{\mathrm{T}}\left(B_{01}^{\mathrm{T}}\right)^{-1}, O_{n-m, n-m}\right)+\left(O_{n-m, m}, E_{n-m}\right)=\left(-B_{02}^{\mathrm{T}}\left(B_{01}^{\mathrm{T}}\right)^{-1}, E_{n-m}\right)=\hat{B}_{0},
$$

we have

$$
(\psi(0), \varphi(0))_{n}=\left(\bar{B}_{0} \psi(0), B_{0} \varphi(0)\right)_{m}+\left(\hat{B}_{0} \psi(0), \tilde{B}_{0} \varphi(0)\right)_{n-m} .
$$

Analogously

$$
(\psi(T), \varphi(T))_{n}=\left(\bar{B}_{1} \psi(T), B_{1} \varphi(T)\right)_{n-m}+\left(\hat{B}_{1} \psi(T), \tilde{B}_{1} \varphi(T)\right)_{m} .
$$

These two equalities yield representation (0.11); using the latter and (0.10), we obtain

$$
\begin{aligned}
& \int_{0}^{T}(L \varphi(t), \psi(t))_{n} \mathrm{~d} t=\left(\bar{B}_{1} \psi(T), B_{1} \varphi(T)\right)_{n-m}+\left(\hat{B}_{1} \psi(T), \tilde{B}_{1} \varphi(T)\right)_{m} \\
& -\left(\bar{B}_{0} \psi(0), B_{0} \varphi(0)\right)_{m}-\left(\hat{B}_{0} \psi(0), \tilde{B}_{0} \varphi(0)\right)_{n-m}+\int_{0}^{T}\left(\varphi(t), L^{*} \psi(t)\right)_{n} .
\end{aligned}
$$

In order to write the sum of the first four terms on the right-hand side of (0.12) in a scalar form, introduce the following notations:

$$
\begin{aligned}
& \left(\begin{array}{c}
U_{n+1}(\varphi) \\
\vdots \\
U_{2 n-m}(\varphi)
\end{array}\right):=\tilde{B}_{0} \varphi(0)=\left(\begin{array}{c}
\sum_{q=1}^{n} \tilde{b}_{1 q}^{(0)} \varphi_{q}(0) \\
\vdots \\
\sum_{q=1}^{n} \tilde{b}_{n-m, q}^{(0)} \varphi_{q}(0)
\end{array}\right), \\
& \left(\begin{array}{c}
U_{2 n-m+1}(\varphi) \\
\vdots \\
U_{2 n}(\varphi)
\end{array}\right):=\tilde{B}_{1} \varphi(T)=\left(\begin{array}{c}
\sum_{q=1}^{n} \tilde{b}_{1 q}^{(1)} \varphi_{q}(T) \\
\vdots \\
\sum_{q=1}^{n} \tilde{b}_{m, q}^{(1)} \varphi_{q}(T)
\end{array}\right), \\
& \left(\begin{array}{c}
V_{2 n}(\psi) \\
\vdots \\
V_{2 n-m+1}(\psi)
\end{array}\right):=\bar{B}_{0} \psi(0)=\left(\begin{array}{c}
\sum_{q=1}^{n} \bar{b}_{1 q}^{(0)} \psi_{q}(0) \\
\vdots \\
\sum_{q=1}^{n} \bar{b}_{m, q}^{(0)} \psi_{q}(0)
\end{array}\right), \\
& \left(\begin{array}{c}
V_{2 n-m}(\psi) \\
\vdots \\
V_{n+1}(\psi)
\end{array}\right):=\bar{B}_{1} \psi(T)=\left(\begin{array}{c}
\sum_{q=1}^{n} \bar{b}_{1 q}^{(1)} \psi_{q}(T) \\
\vdots \\
\sum_{q=1}^{n} \bar{b}_{n-m, q}^{(1)} \psi_{q}(T)
\end{array}\right) \text {, } \\
& \left(\begin{array}{c}
V_{n}(\psi) \\
\vdots \\
V_{m+1}(\psi)
\end{array}\right):=\hat{B}_{0} \psi(0)=\left(\begin{array}{c}
\sum_{q=1}^{n} \hat{b}_{1 q}^{(0)} \psi_{q}(0) \\
\vdots \\
\sum_{q=1}^{n} \hat{b}_{n-m, q}^{(0)} \psi_{q}(0)
\end{array}\right) \text {, } \\
& \left(\begin{array}{c}
V_{m}(\psi) \\
\vdots \\
V_{1}(\psi)
\end{array}\right):=\hat{B}_{1} \psi(T)=\left(\begin{array}{c}
\sum_{q=1}^{n} \hat{b}_{1 q}^{(1)} \psi_{q}(T) \\
\vdots \\
\sum_{q=1}^{n} \hat{b}_{m, q}^{(1)} \psi_{q}(T)
\end{array}\right) .
\end{aligned}
$$

Then the Equality (0.12) can be written as 


$$
\begin{aligned}
\int_{0}^{T} & (L \varphi(t), \psi(t))_{n} \mathrm{~d} t-\int_{0}^{T}\left(\varphi(t), L^{*} \psi(t)\right)_{n} \\
= & -U_{1}(\varphi) V_{2 n}(\psi)-U_{2}(\varphi) V_{2 n-1}(\psi)-\cdots-U_{m}(\varphi) V_{2 n-m+1}(\psi) \\
& +U_{m+1}(\varphi) V_{2 n-m}(\psi)+U_{m+2}(\varphi) V_{2 n-m-1}(\psi)+\cdots+U_{n}(\varphi) V_{n+1}(\psi) \\
& -U_{n+1}(\varphi) V_{n}(\psi)-U_{n+2}(\varphi) V_{n-1}(\psi)-\cdots-U_{2 n-m}(\varphi) V_{m+1}(\psi) \\
& +U_{2 n-m+1}(\varphi) V_{m}(\psi)+U_{2 n-m+2}(\varphi) V_{m-1}(\psi)+\cdots+U_{2 n}(\varphi) V_{1}(\psi) .
\end{aligned}
$$

Now we can introduce the notion of the adjoint BVP.

Definition 2.1 The homogeneous BVP

$$
\begin{gathered}
L^{*} \psi(t)=0, \quad t \in(0, T), \\
\hat{B}_{0} \psi(0)=0, \quad \hat{B}_{1} \psi(T)=0,
\end{gathered}
$$

is called adjoint to homogeneous BVP (0.3), (0.4).

Definition 2.2 The inhomogeneous BVP

$$
\begin{gathered}
L^{*} \psi(t)=\tilde{f}(t), \quad t \in(0, T), \\
\hat{B}_{0} \psi(0)=\tilde{f}_{0}, \quad \hat{B}_{1} \psi(T)=\tilde{f}_{1},
\end{gathered}
$$

where $\tilde{f} \in\left(L^{2}(0, T)\right)^{n}, \quad \tilde{f}_{0} \in \mathbb{R}^{n-m}, \quad \tilde{f}_{1} \in \mathbb{R}^{m}$, is called adjoint to inhomogeneous BVP (0.1), (0.2).

The results contained in [13] [14] imply that the following statement is valid (for more detailed explanations see [9], pp. 9-11).

Theorem 2.1 If homogeneous BVP (0.3), (0.4) has only the trivial solution, then the corresponding adjoint BVP (0.20), (0.21) also has only the trivial solution and inhomogeneous BVP (0.22), (0.23) has one and only one solution $\psi \in\left(H^{1}(0, T)\right)^{n}$ at any $\tilde{f}(t) \in\left(L^{2}(0, T)\right)^{n}, \quad \tilde{f}_{0} \in \mathbb{R}^{n-m}, \quad \tilde{f}_{1} \in \mathbb{R}^{m}$.

\section{Statement of the Minimax Estimation Problem and Its Reduction to an Optimal Control Problem}

Let a vector-function

$$
y(t)=H(t) \varphi(t)+\xi(t),
$$

with the values from the space $\mathbb{R}^{l}$ be observed on an interval $(\alpha, \beta) \subseteq(0, T)$; here $H(t)$ is an $l \times n$ matrix with the entries that are continuous functions on $[\alpha, \beta]$, and $\xi(t) \in \mathbb{R}^{l}$ is an unknown random vector process whose realizations enter observations (0.24).

Denote by $V$ the set of random vector processes $\tilde{\xi}(t)$ with zero expectation $\mathbb{E} \tilde{\xi}(t)$ and second moments $\mathbb{E} \tilde{\xi}(t)^{2}$ integrable on $(\alpha, \beta)$ such that their correlation matrices $\tilde{R}(t, s)=\mathbb{E} \tilde{\xi}(t) \tilde{\xi}^{\mathrm{T}}(s)$ satisfy the inequality

$$
\left\{\tilde{R}: \int_{\alpha}^{\beta} \operatorname{Sp}[Q(t) \tilde{R}(t, t)] \mathrm{d} t \leq \varepsilon_{1}\right\},
$$

where $Q(t)$ is a positive definite matrix of dimension $l \times l$, the entries of $Q(t)$ and $Q^{-1}(t)$ are continuous on $[\alpha, \beta], \varepsilon_{1}$ is a given positive number, $\mathrm{Sp} B:=\sum_{i=1}^{l} b_{i i}$ denotes the trace of the matrix $B=\left\{b_{i j}\right\}_{i, j=1}^{l}$.

$$
\begin{aligned}
G=\left\{\tilde{F}:=\left(\tilde{f}_{0}, \tilde{f}_{1}, \tilde{f}(\cdot)\right) \in \mathbb{R}^{m} \times \mathbb{R}^{n-m} \times\left(L^{2}(0, T)\right)^{n}:\left(Q_{0}\left(\tilde{f}_{0}-f_{0}^{(0)}\right), \tilde{f}_{0}-f_{0}^{(0)}\right)_{m}\right. \\
\left.+\left(Q_{1}\left(\tilde{f}_{1}-f_{1}^{(0)}\right), \tilde{f}_{1}-f_{1}^{(0)}\right)_{n-m}+\int_{0}^{T}\left(Q_{2}(t)\left(\tilde{f}(t)-f^{(0)}(t)\right), \tilde{f}(t)-f^{(0)}(t)\right)_{n} \mathrm{~d} t \leq \varepsilon_{2}\right\},
\end{aligned}
$$

where $f_{0}^{(0)} \in \mathbb{R}^{m}, \quad f_{1}^{(0)} \in \mathbb{R}^{n-m}$ are given vectors; $f^{(0)} \in\left(L^{2}(0, T)\right)^{n}$ is a given vector-function; $Q_{0}, Q_{1}$, and $Q_{2}(t)$ are positive definite matrices of dimensions $m \times m,(n-m) \times(n-m)$, and $n \times n$, respectively, the entries of $Q_{2}(t)$ and $Q_{2}^{-1}(t)$ are continuous on $[0, T], \varepsilon_{2}$ is a given positive number.

Assume that the right-hand sides $f(\cdot), \quad f_{0}$, and $f_{1}$ of Equation (0.1) and boundary conditions (0.2) are 
not known exactly and it is known only that the element $F:=\left(f_{0}, f_{1}, f(\cdot)\right)$ belongs to a set $G$ and, additionally, $\xi(t) \in V$. Further we also will assume, without loss of generality, that in (0.25) and (0.26) $\varepsilon_{1}=\varepsilon_{2}=1$.

Let a vector-function $\varphi(t)$ be a solution to BVP (0.1), (0.2).

We will look for an estimation of the inner product

$$
(a, \varphi(s))_{n}
$$

in the class of estimates linear with respect to observations that have the form

$$
\begin{aligned}
\overline{(a, \varphi(s))_{n}} & =\int_{\alpha}^{\beta}(u(t), y(t))_{l} \mathrm{~d} t+c \\
& =\int_{\alpha}^{s}\left(u_{1}(t), y(t)\right)_{l} \mathrm{~d} t+\int_{s}^{\beta}\left(u_{2}(t), y(t)\right)_{I} \mathrm{~d} t+c,
\end{aligned}
$$

where $s \in(\alpha, \beta)^{1}$ and $a$ is a vector belonging to $\mathbb{R}^{n}, \quad u \in\left(L^{2}(\alpha, \beta)\right)^{l}, \quad u_{1}=\left.u\right|_{(\alpha, s)}, \quad u_{2}=\left.u\right|_{(s, T)}$, and $c$ is certain constant. Then $u=\left(u_{1}, u_{2}\right) \in H:=\left(L^{2}(\alpha, s)\right)^{l} \times\left(L^{2}(s, \beta)\right)^{l}=\left(L^{2}(\alpha, \beta)\right)^{l}$.

Definition 3.1 An estimate

$$
\begin{aligned}
\overline{\overline{(a, \varphi(s))_{n}}} & =\int_{\alpha}^{\beta}(\hat{u}(t), y(t))_{l} \mathrm{~d} t+\hat{c} \\
& =\int_{\alpha}^{s}\left(\hat{u}_{1}(t), y(t)\right)_{l} \mathrm{~d} t+\int_{s}^{\beta}\left(\hat{u}_{2}(t), y(t)\right)_{l} \mathrm{~d} t+\hat{c}
\end{aligned}
$$

for which vector-function $\hat{u}(t)=\left(\hat{u}_{1}(t), \hat{u}_{2}(t)\right)$ and constant $\hat{c}$ are determined from the condition

$$
\inf _{u \in H, c \in \mathbb{R}} \sigma(u, c)=\sigma(\hat{u}, \hat{c}) \text {, }
$$

where

$$
\begin{gathered}
\sigma(u, c):=\sup _{\tilde{F} \in G, \tilde{\xi} \in V} \mathbb{E}\left|(a, \tilde{\varphi}(s))_{n}-\overline{(a, \tilde{\varphi}(s))_{n}}\right|^{2}, \\
\tilde{\varphi} \text { is a solution to BVP (0.1), (0.2) at } f(t)=\tilde{f}(t), \quad f_{0}=\tilde{f}_{0}, \quad f_{1}=\tilde{f}_{1}, \quad \text { and } \\
\overline{(a, \tilde{\varphi}(s))_{n}}=\int_{\alpha}^{s}\left(u_{1}(t), \tilde{y}(t)\right)_{l} \mathrm{~d} t+\int_{s}^{\beta}\left(u_{2}(t), \tilde{y}(t)\right)_{l} \mathrm{~d} t+c, \\
\tilde{y}(t)=H(t) \tilde{\varphi}(t)+\tilde{\xi}(t),
\end{gathered}
$$

will be called a minimax mean square estimate of inner product $(a, \varphi(s))_{n}$. The quantity

$$
\sigma=[\sigma(\hat{u}, \hat{c})]^{1 / 2}
$$

will be called an error of the minimax estimation.

We see that the minimax mean square estimate of inner product $(a, \varphi(s))_{n}$ is an estimate at which the maximum mean square estimation error calculated for the worst realization of perturbations attains its minimum.

We will show that solution to the minimax estimation problem is reduced to the solution of a certain optimal control problem.

For every fixed $u:=\left(u_{1}, u_{2}\right) \in H$ introduce vector-functions $z_{1}(; ; u) \in\left(H^{1}(0, \alpha)\right)^{n}, \quad z_{2}(; ; u) \in\left(H^{1}(\alpha, s)\right)^{n}$, $z_{3}(; ; u) \in\left(H^{1}(s, \beta)\right)^{n}$, and $z_{4}(; \cdot u) \in\left(H^{1}(\beta, T)\right)^{n}$ as a solution to the following BVP:

$$
\begin{aligned}
& L^{*} z_{1}(t ; u)=0, \quad 0<t<\alpha, \quad \hat{B}_{0} z_{1}(0 ; u)=0, \\
& L^{*} z_{2}(t ; u)=-H^{\mathrm{T}}(t) u_{1}(t), \quad \alpha<t<s, \quad z_{2}(\alpha ; u)=z_{1}(\alpha ; u), \\
& L^{*} z_{3}(t ; u)=-H^{\mathrm{T}}(t) u_{2}(t), \quad s<t<\beta, \quad z_{3}(s ; u)=z_{2}(s ; u)-a, \\
& L^{*} z_{4}(t ; u)=0, \quad \beta<t<T, \quad z_{4}(\beta ; u)=z_{3}(\beta ; u), \quad \hat{B}_{1} z_{4}(T ; u)=0 .
\end{aligned}
$$

Lemma 3.1 Determination of the minimax mean square estimate of inner product $(a, \varphi(s))_{n}$. is equivalent

$\overline{{ }^{1} \text { If } s \in(0, T) \backslash(\alpha, \beta) \text { then the minimax estimation }}$ problem can be solved in a similar manner but somewhat simpler. 
to the problem of optimal control of the system described by BVP $(0.29)$ with the cost function

$$
\begin{aligned}
I(u)= & \left(Q_{0}^{-1} \bar{B}_{0} z_{1}(0 ; u), \bar{B}_{0} z_{1}(0 ; u)\right)_{m}+\left(Q_{1}^{-1} \bar{B}_{1} z_{4}(T ; u), \bar{B}_{1} z_{4}(T ; u)\right)_{n-m} \\
& +\int_{0}^{\alpha}\left(Q_{2}^{-1}(t) z_{1}(t ; u), z_{1}(t ; u)\right)_{n} \mathrm{~d} t+\int_{\alpha}^{s}\left(Q_{2}^{-1}(t) z_{2}(t ; u), z_{2}(t ; u)\right)_{n} \mathrm{~d} t \\
& +\int_{s}^{\beta}\left(Q_{2}^{-1}(t) z_{3}(t ; u), z_{3}(t ; u)\right)_{n} \mathrm{~d} t+\int_{\beta}^{T}\left(Q_{2}^{-1}(t) z_{4}(t ; u), z_{4}(t ; u)\right)_{n} \mathrm{~d} t \\
& +\int_{\alpha}^{s}\left(Q^{-1}(t) u_{1}(t), u_{1}(t)\right)_{l} \mathrm{~d} t+\int_{s}^{\beta}\left(Q^{-1}(t) u_{2}(t), u_{2}(t)\right)_{l} \mathrm{~d} t .
\end{aligned}
$$

Proof. Show first that BVP (0.29) is uniquely solvable under the condition that functions $u_{1}(t)$ and $u_{2}(t)$ belong, respectively, to the spaces $\left(L^{2}(\alpha, s)\right)^{l}$ and $\left(L^{2}(s, \beta)\right)^{l}$.

Since homogeneous BVP (0.3), (0.4) has only the trivial solution, the BVP

$$
L^{*} \psi(t)=g(t), 0<t<T, \hat{B}_{0} \psi(0)=0, \hat{B}_{1} \psi(T)=0
$$

has, in line with Theorem 2.1, the unique solution for any right-hand side, in particular, at

$$
g(t)=g(t ; u)= \begin{cases}0, & 0<t<\alpha ; \\ -H^{\mathrm{T}}(t) u_{1}(t), & \alpha<t<s ; \\ -H^{\mathrm{T}}(t) u_{2}(t), & s<t<\beta ; \\ 0, & \beta<t<T .\end{cases}
$$

Denote this solution by $\bar{z}(t ; u)$ and its restrictions on intervals $(0, \alpha), \quad(\alpha, s), \quad(s, \beta)$, and $(\beta, T)$ by $\bar{z}_{1}(t ; u), \quad \bar{z}_{2}(t ; u), \quad \bar{z}_{3}(t ; u)$, and $\bar{z}_{4}(t ; u)$, respectively. Note that function $\bar{z}(t ; u)$ is absolutely continuous on $[0, T]$ (see [15]).

Let us show that the problem

$$
\begin{array}{ll}
L^{*} \overline{\bar{Z}}^{(1)}(t)=0, \quad 0<t<\alpha, & \hat{B}_{0} \overline{\bar{Z}}^{(1)}(0)=0, \\
L^{*} \overline{\bar{Z}}^{(2)}(t)=0, \quad \alpha<t<s, & \overline{\bar{Z}}^{(2)}(\alpha ; u)=\overline{\bar{Z}}^{(1)}(\alpha ; u), \\
L^{*} \overline{\bar{Z}}^{(3)}(t)=0, \quad s<t<\beta, & \overline{\bar{Z}}^{(3)}(s ; u)=\overline{\bar{Z}}^{(2)}(s)-a, \\
L^{*} \overline{\bar{Z}}^{(4)}(t)=0, \quad \beta<t<T, & \overline{\bar{Z}}^{(4)}(\beta)=\overline{\bar{Z}}^{(3)}(\beta), \quad \hat{B}_{1} \overline{\bar{Z}}^{(4)}(T)=0
\end{array}
$$

has one and only one solution at any vector $a \in \mathbb{R}^{n}$.

Denote by $\overline{\bar{Z}}_{i}^{(j)}(t), \quad i=\overline{1, n}, \quad j=\overline{1,4}$, the coordinates of vector-function $\overline{\bar{Z}}^{(j)}(t), \quad j=\overline{1,4}$. Let $y_{i k}(t)$, $i, k=\overline{1, n}$ be the fundamental system of solutions of the equation system $L^{*} z(t)=0$ on $[0, T]$. Then we can represent functions $\overline{\bar{z}}_{i}^{(j)}(t), \quad i=\overline{1, n}, \quad j=\overline{1,4}$, in the form

$$
\overline{\bar{z}}_{i}^{(j)}(t)=\sum_{k=1}^{n} c_{k}^{(j)} y_{i k}(t)
$$

where $c_{k}^{(j)}$ are constants. Taking into account the boundary conditions at the points $t=0, T$ and transmission conditions at $t=\alpha, s, \beta$ in (0.33), we see that the solution to BVP (0.33) is equivalent to the solution of the following linear equation system with $4 n$ unknowns $c_{k}^{(j)}, \quad k=\overline{1, n}, \quad j=\overline{1,4}$ :

$$
\begin{gathered}
\sum_{k=1}^{n} a_{i k}^{0} c_{k}^{(1)}=0, \quad i=\overline{1, n-m}, \\
\sum_{k=1}^{n} y_{i k}(\alpha)\left(c_{k}^{(1)}-c_{k}^{(2)}\right)=0, \quad i=\overline{1, n}, \\
\sum_{k=1}^{n} y_{i k}(s)\left(c_{k}^{(2)}-c_{k}^{(3)}\right)=a_{i}, \quad i=\overline{1, n},
\end{gathered}
$$




$$
\begin{gathered}
\sum_{k=1}^{n} y_{i k}(\beta)\left(c_{k}^{(3)}-c_{k}^{(4)}\right)=0, \quad i=\overline{1, n}, \\
\sum_{k=1}^{n} a_{i k}^{1} c_{k}^{(4)}=0, \quad i=\overline{1, m},
\end{gathered}
$$

where

$$
\begin{gathered}
a_{i k}^{0}=\sum_{r=1}^{n} \hat{b}_{i r}^{(0)} y_{r k}(0), \quad i=\overline{1, n-m}, \quad k=\overline{1, n}, \\
a_{i k}^{1}=\sum_{s=1}^{n} \hat{b}_{i s}^{(1)} y_{s k}(T), \quad i=\overline{1, m}, \quad k=\overline{1, n},
\end{gathered}
$$

$a_{i}, \quad i=\overline{1, n}$, denote the coordinates of vector $a$, and $\hat{b}_{i r}^{(0)}, \quad i=\overline{1, n-m}, \quad r=\overline{1, n}$, and $\hat{b}_{i s}^{(1)}, \quad i=\overline{1, m}$, $s=1, n$, denote the entries of matrices $\hat{B}_{0}$ and $\hat{B}_{1}$, respectively.

Show that system (0.34)-(0.38) is uniquely solvable at any vector $a \in \mathbb{R}^{n}$. To this end, note that homogeneous system (0.34)-(0.38) (at $a=0$ ) has only the trivial solution.

Indeed, setting $a=0$ in Equations (0.35) and (0.36), taking into account (0.37) and the fact that $\operatorname{det}\left\{y_{i k}(\alpha)\right\}_{i, k=1}^{n} \neq 0$, $\operatorname{det}\left\{y_{i k}(s)\right\}_{i, k=1}^{n} \neq 0$, and $\operatorname{det}\left\{y_{i k}(\beta)\right\}_{i, k=1}^{n} \neq 0$ because $y_{i k}(t), \quad i, k=\overline{1, n}$ is the fundamental system of solutions of the equation system $L^{*} z(t)=0$ on $[0, T]$, we obtain

$$
c_{k}^{(1)}=c_{k}^{(2)}=c_{k}^{(3)}=c_{k}^{(4)}=: c_{k} .
$$

Coefficients $\quad c_{k}$ satisfy Equations (0.34) and (0.38); therefore vector-function $\psi(t)$ with the components $\psi_{i}(t)=\sum_{k=1}^{n} c_{k} y_{i k}(t), \quad i=\overline{1, n}$ is a solution to conjugate BVP $(0.20),(0.21)$ which has only the trivial solution $\psi(t) \equiv 0$ on $[0, T]$ by Theorem 2.1. This implies $c_{k}=0$, so the homogeneous linear equation system (0.34)(0.38) (at $a=0$ ) has only the trivial solution. Consequently, system (0.34)-(0.38) and therefore BVP (0.33) which is equivalent to this system are uniquely solvable at any vector $a \in \mathbb{R}^{n}$. Then vector-functions $z_{i}(t ; u)=\bar{z}_{i}(t ; u)+\overline{\bar{z}}^{(i)}(t), \quad i=\overline{1,4}$, form the unique solution to BVP $(0.29)$.

Show next that the determination of the minimax estimate of inner product $(a, \varphi(s))_{n}$ is equivalent to the problem of optimal control of the system described by BVP (0.29) with the cost function (0.30).

Using the second and third equations in (0.29) and the fact that $\tilde{\varphi}$ is a solution to BVP $(0.1),(0.2)$ at $f(t)=\tilde{f}(t), \quad f_{0}=\tilde{f}_{0}$, and $f_{1}=\tilde{f}_{1}$, we easily obtain the relationships

$$
\begin{aligned}
& -\int_{\alpha}^{s}\left(H^{\mathrm{T}}(t) u_{1}(t), \tilde{\varphi}(t)\right)_{n} \mathrm{~d} t=\left(z_{2}(\alpha ; u), \tilde{\varphi}(\alpha)\right)_{n}-\left(z_{2}(s ; u), \tilde{\varphi}(s)\right)_{n}+\int_{\alpha}^{s}\left(z_{2}(t ; u), \tilde{f}(t)\right)_{n} \mathrm{~d} t, \\
& -\int_{s}^{\beta}\left(H^{T}(t) u_{2}(t), \tilde{\varphi}(t)\right)_{n} \mathrm{~d} t=\left(z_{3}(s ; u), \tilde{\varphi}(s)\right)_{n}-\left(z_{3}(\beta ; u), \tilde{\varphi}(\beta)\right)_{n}+\int_{s}^{\beta}\left(z_{3}(t ; u), \tilde{f}(t)\right)_{n} \mathrm{~d} t .
\end{aligned}
$$

Taking into account the equalities

$$
\begin{gathered}
z_{2}(\alpha ; u)=z_{1}(\alpha ; u), \quad z_{2}(s ; u)-z_{3}(s ; u)=a, \quad z_{3}(\beta ; u)=z_{4}(\beta ; u), \\
\left(z_{1}(\alpha ; u), \tilde{\varphi}(\alpha)\right)_{n}=\int_{0}^{\alpha} \mathrm{d}\left(z_{1}(t ; u), \tilde{\varphi}(t)\right)_{n}+\left(z_{1}(0 ; u), \tilde{\varphi}(0)\right)_{n}, \\
\left(z_{4}(\beta ; u), \tilde{\varphi}(\beta)\right)_{n}=-\int_{\beta}^{T} \mathrm{~d}\left(z_{4}(t ; u), \tilde{\varphi}(t)\right)_{n}+\left(z_{4}(T ; u), \tilde{\varphi}(T)\right)_{n},
\end{gathered}
$$

and that

$$
\begin{gathered}
\left(z_{1}(0 ; u), \tilde{\varphi}(0)\right)_{n}=\left(\bar{B}_{0} z_{1}(0 ; u), B_{0} \tilde{\varphi}(0)\right)_{m}+\left(\hat{B}_{0} z_{1}(0 ; u), \tilde{B}_{0} \tilde{\varphi}(0)\right)_{n-m}=\left(\bar{B}_{0} z_{1}(0 ; u), \tilde{f}_{0}\right)_{m}, \\
\left(z_{4}(T ; u), \tilde{\varphi}(T)\right)_{n}=\left(\bar{B}_{1} z_{4}(T ; u), B_{1} \tilde{\varphi}(T)\right)_{n-m}+\left(\hat{B}_{1} z_{4}(T ; u), \tilde{B} \tilde{\varphi}_{1}(T)\right)_{m}=\left(\bar{B}_{1} z_{4}(T ; u), \tilde{f}_{1}\right)_{n-m},
\end{gathered}
$$

(we refer to the reasoning on p. 4) we obtain 


$$
\begin{aligned}
(a, \tilde{\varphi}(s))_{n}-(\widehat{a, \tilde{\varphi}(s)})_{n}=\left(z_{2}(s ; u), \tilde{\varphi}(s)\right)_{n}-\left(z_{3}(s ; u), \tilde{\varphi}(s)\right)_{n}-(\widehat{a, \tilde{\varphi}(s)})_{n} \\
=\left(z_{2}(\alpha ; u), \tilde{\varphi}(\alpha)\right)_{n}+\int_{\alpha}^{s}\left(H^{\mathrm{T}}(t) u_{1}(t), \tilde{\varphi}(t)\right)_{n} \mathrm{~d} t+\int_{\alpha}^{s}\left(z_{2}(t ; u), \tilde{f}(t)\right)_{n} \mathrm{~d} t \\
\quad-\left(z_{3}(\beta ; u), \tilde{\varphi}(\beta)\right)_{n}+\int_{s}^{\beta}\left(H^{\mathrm{T}}(t) u_{2}(t), \tilde{\varphi}(t)\right)_{n} \mathrm{~d} t+\int_{s}^{\beta}\left(z_{3}(t ; u), \tilde{f}(t)\right)_{n} \mathrm{~d} t \\
\quad-\int_{\alpha}^{s}\left(u_{1}(t), \tilde{y}(t)\right)_{l} \mathrm{~d} t-\int_{s}^{\beta}\left(u_{2}(t), \tilde{y}(t)\right)_{l} \mathrm{~d} t-c \\
=\int_{0}^{\alpha} \mathrm{d}\left(z_{1}(t ; u), \tilde{\varphi}(t)\right)_{n}+\left(z_{1}(0 ; u), \tilde{\varphi}(0)\right)_{n}+\int_{\alpha}^{s}\left(H^{\mathrm{T}}(t) u_{1}(t), \tilde{\varphi}(t)\right)_{n} \mathrm{~d} t \\
\quad+\int_{\alpha}^{s}\left(z_{2}(t ; u), \tilde{f}(t)\right)_{n} \mathrm{~d} t+\int_{\beta}^{T} \mathrm{~d}\left(z_{4}(t ; u), \tilde{\varphi}(t)\right)_{n}-\left(z_{4}(T ; u), \tilde{\varphi}(T)\right)_{n} \\
\quad+\int_{s}^{\beta}\left(H^{\mathrm{T}}(t) u_{2}(t), \tilde{\varphi}(t)\right)_{n} \mathrm{~d} t+\int_{s}^{\beta}\left(z_{3}(t ; u), \tilde{f}(t)\right)_{n} \mathrm{~d} t-\int_{\alpha}^{s}\left(H^{\mathrm{T}}(t) u_{1}(t), \tilde{\varphi}(t)\right)_{n} \mathrm{~d} t \\
\quad-\int_{\alpha}^{s}\left(u_{1}(t), \tilde{\xi}(t)\right)_{l} \mathrm{~d} t-\int_{s}^{\beta}\left(H^{\mathrm{T}}(t) u_{2}(t), \tilde{\varphi}(t)\right)_{n} \mathrm{~d} t-\int_{s}^{\beta}\left(u_{2}(t), \tilde{\xi}(t)\right)_{l} \mathrm{~d} t-c \\
=\int_{0}^{\alpha}\left(\frac{\mathrm{d} z_{1}(t ; u)}{\mathrm{d} t}, \tilde{\varphi}(t)\right)_{n} \mathrm{~d} t+\int_{0}^{\alpha}\left(\frac{\mathrm{d} \tilde{\varphi}(t)}{\mathrm{d} t}, z_{1}(t ; u)\right)_{n} \mathrm{~d} t+\left(\bar{B}_{0} z_{1}(0 ; u), \tilde{f}_{0}\right)_{m} \\
\quad+\int_{\alpha}^{s}\left(z_{2}(t ; u), \tilde{f}(t)\right)_{n} \mathrm{~d} t+\int_{\beta}^{T}\left(\frac{\mathrm{d} z_{4}(t ; u)}{\mathrm{d} t}, \tilde{\varphi}(t)\right)_{n} \mathrm{~d} t+\int_{\beta}^{T}\left(\frac{\mathrm{d} \tilde{\varphi}(t)}{\mathrm{d} t}, z_{4}(t ; u)\right)_{n} \mathrm{~d} t \\
\quad-\left(\bar{B}_{1} z_{4}(T ; u), \tilde{f_{1}}\right)_{n-m}+\int_{s}^{\beta}\left(z_{3}(t ; u), \tilde{f}(t)\right)_{n} \mathrm{~d} t-\int_{\alpha}^{s}\left(u_{1}(t), \tilde{\xi}(t)\right)_{l} \mathrm{~d} t-\int_{s}^{\beta}\left(u_{2}(t), \tilde{\xi}(t)\right)_{l} \mathrm{~d} t-c .
\end{aligned}
$$

Taking into notice that

$$
\begin{gathered}
\frac{\mathrm{d} z_{1}(t ; u)}{\mathrm{d} t}=A^{\mathrm{T}}(t) z_{1}(t ; u) \quad \text { on }(0, \alpha), \quad \frac{\mathrm{d} z_{4}(t ; u)}{\mathrm{d} t}=A^{\mathrm{T}}(t) z_{4}(t ; u) \quad \text { on }(\beta, T) \\
\frac{\mathrm{d} \tilde{\varphi}(t)}{\mathrm{d} t}=\tilde{f}(t)-A(t) \tilde{\varphi}(t) \quad \text { on }(0, T),
\end{gathered}
$$

and therefore

$$
\begin{gathered}
\int_{0}^{\alpha}\left(\frac{\mathrm{d} z_{1}(t ; u)}{\mathrm{d} t}, \tilde{\varphi}(t)\right)_{n} \mathrm{~d} t+\int_{0}^{\alpha}\left(\frac{\mathrm{d} \tilde{\varphi}(t)}{\mathrm{d} t}, z_{1}(t ; u)\right)_{n} \mathrm{~d} t \\
=\int_{0}^{\alpha}\left(A^{T}(t) z_{1}(t ; u), \tilde{\varphi}(t)\right)_{n} \mathrm{~d} t+\int_{0}^{\alpha}\left(z_{1}(t ; u), \tilde{f}(t)-A(t) \tilde{\varphi}(t)\right)_{n} \mathrm{~d} t \\
=\int_{0}^{\alpha}\left(z_{1}(t ; u), \tilde{f}(t)\right)_{n} \mathrm{~d} t, \\
\int_{\beta}^{T}\left(\frac{\mathrm{d} z_{4}(t ; u)}{\mathrm{d} t}, \tilde{\varphi}(t)\right)_{n} \mathrm{~d} t+\int_{\beta}^{T}\left(\frac{\mathrm{d} \tilde{\varphi}(t)}{\mathrm{d} t}, z_{4}(t ; u)\right)_{n} \mathrm{~d} t=\int_{\beta}^{T}\left(z_{4}(t ; u), \tilde{f}(t)\right)_{n} \mathrm{~d} t,
\end{gathered}
$$

we use the last equality to obtain

$$
\begin{aligned}
& (a, \tilde{\varphi}(s))_{n}-(\widehat{a, \tilde{\varphi}(s)})_{n}=\left(\bar{B}_{0} z_{1}(0 ; u), \tilde{f}_{0}\right)_{m}+\int_{0}^{T}(z(t ; u), \tilde{f}(t))_{n} \mathrm{~d} t \\
& -\left(\bar{B}_{1} z_{4}(T ; u), \tilde{f}_{1}\right)_{n-m}-\int_{\alpha}^{s}\left(u_{1}(t), \tilde{\xi}(t)\right)_{l} \mathrm{~d} t-\int_{s}^{\beta}\left(u_{2}(t), \tilde{\xi}(t)\right)_{l} \mathrm{~d} t-c=: \eta,
\end{aligned}
$$

where

$$
z(t ; u)= \begin{cases}z_{1}(t ; u), & 0 \leq t \leq \alpha ; \\ z_{2}(t ; u), & \alpha<t<s ; \\ z_{3}(t ; u), & s<t<\beta ; \\ z_{4}(t ; u), & \beta \leq t \leq T .\end{cases}
$$


Recalling that $\tilde{\xi}(t)$ is a vector process with zero expectation, we use condition (0.25) and the known relationship $D \eta=\mathbb{E} \eta^{2}-(\mathbb{E} \eta)^{2}$ that couples the dispersion $D \eta:=\mathbb{E}[\eta-\mathbb{E} \eta]^{2}$ of random variable $\eta$ with its expectation $\mathbb{E} \eta$, to obtain

$$
\begin{gathered}
\mathbb{E} \eta=\left(\bar{B}_{0} z_{1}(0 ; u), \tilde{f}_{0}\right)_{m}+\int_{0}^{T}(z(t ; u), \tilde{f}(t))_{n} \mathrm{~d} t-\left(\bar{B}_{1} z_{4}(T ; u), \tilde{f}_{1}\right)_{n-m}-c, \\
\eta-\mathbb{E} \eta=-\int_{\alpha}^{s}\left(u_{1}(t), \tilde{\xi}(t)\right)_{l} \mathrm{~d} t-\int_{s}^{\beta}\left(u_{2}(t), \tilde{\xi}(t)\right)_{l} \mathrm{~d} t \\
D \eta=\mathbb{E}[\eta-\mathbb{E} \eta]^{2}=\mathbb{E}\left[\int_{\alpha}^{s}\left(u_{1}(t), \tilde{\xi}(t)\right)_{l} \mathrm{~d} t+\int_{s}^{\beta}\left(u_{2}(t), \tilde{\xi}(t)\right)_{l} \mathrm{~d} t\right]^{2}, \\
\mathbb{E} \eta^{2}=D \eta+(\mathbb{E} \eta)^{2}=\mathbb{E}\left[\int_{\alpha}^{s}\left(u_{1}(t), \tilde{\xi}(t)\right)_{l} \mathrm{~d} t+\int_{s}^{\beta}\left(u_{2}(t), \tilde{\xi}(t)\right)_{l} \mathrm{~d} t\right]^{2} \\
+\left[\left(\bar{B}_{0} z_{1}(0 ; u), \tilde{f}_{0}\right)_{m}+\int_{0}^{T} z((t ; u), \tilde{f}(t))_{n} \mathrm{~d} t-\left(\bar{B}_{1} z_{4}(T ; u), \tilde{f}_{1}\right)_{n-m}-c\right]^{2},
\end{gathered}
$$

which yields

$$
\begin{aligned}
& \inf _{c \in \mathbb{R}^{1}} \sup _{\tilde{F} \in G, \xi \in V} \mathbb{E}\left[(a, \tilde{\varphi}(s))_{n}-(\widehat{a, \tilde{\varphi}(s)})_{n}\right]^{2} \\
& =\inf _{c \in \mathbb{R}^{\mathbb{R}}} \sup _{\tilde{F} \in G}\left[\left(\bar{B}_{0} z_{1}(0 ; u), \tilde{f}_{0}\right)_{m}+\int_{0}^{T}(z(t ; u), \tilde{f}(t))_{n} \mathrm{~d} t-\left(\bar{B}_{1} z_{4}(T ; u), \tilde{f}_{1}\right)_{n-m}-c\right]^{2} \\
& \quad+\sup _{\tilde{\xi} \in V}\left[\int_{\alpha}^{s}\left(u_{1}(t), \tilde{\xi}(t)\right)_{l} \mathrm{~d} t+\int_{s}^{\beta}\left(u_{2}(t), \tilde{\xi}(t)\right)_{l} \mathrm{~d} t\right]^{2} .
\end{aligned}
$$

In order to calculate the supremum on the right-hand side of $(0.40)$ we apply the generalized CauchyBunyakovsky inequality [16] and write this inequality in the form convenient for further analysis: for any $f_{0}^{(1)}, f_{0}^{(2)} \in R^{m}, f_{1}^{(1)}, f_{1}^{(2)} \in R^{n-m}, f_{1}, f_{2} \in\left(L^{2}(0, T)\right)^{n}$, the generalized Cauchy-Bunyakovsky inequality holds

$$
\begin{aligned}
& \left|\left(f_{0}^{(1)}, f_{0}^{(2)}\right)_{m}+\left(f_{1}^{(1)}, f_{1}^{(2)}\right)_{n-m}+\int_{0}^{T}\left(f_{1}(t), f_{2}(t)\right)_{n} \mathrm{~d} t\right| \\
& \leq\left\{\left(Q_{0}^{-1} f_{0}^{(1)}, f_{0}^{(1)}\right)_{m}+\left(Q_{1}^{-1} f_{1}^{(1)}, f_{1}^{(1)}\right)_{n-m}+\int_{0}^{T}\left(Q_{2}^{-1}(t) f_{1}(t), f_{1}(t)\right)_{n} \mathrm{~d} t\right\}^{\frac{1}{2}} \\
& \quad \times\left\{\left(Q_{0} f_{0}^{(2)}, f_{0}^{(2)}\right)_{m}+\left(Q_{1} f_{1}^{(2)}, f_{1}^{(2)}\right)_{n-m}+\int_{0}^{T}\left(Q_{2}(t) f_{2}(t), f_{2}(t)\right)_{n} \mathrm{~d} t\right\}^{\frac{1}{2}},
\end{aligned}
$$

in which the equality is attained at

$$
f_{0}^{(2)}=\lambda Q_{0}^{-1} f_{0}^{(1)}, \quad f_{1}^{(2)}=\lambda Q_{1}^{-1} f_{1}^{(1)}, \quad f_{2}(t)=\lambda Q_{2}^{-1} f_{1}(t) .
$$

Setting in the generalized Cauchy-Bunyakovsky inequality

$$
\begin{gathered}
f_{0}^{(1)}=\bar{B}_{0} z_{1}(0 ; u), \quad f_{1}^{(1)}=-\bar{B}_{1} z_{4}(T ; u), \quad f_{1}(t)=\tilde{z}(t ; u), \\
f_{0}^{(2)}=\tilde{f}_{0}-f_{0}^{(0)}, \quad f_{1}^{(2)}=\tilde{f}_{1}-f_{1}^{(0)}, \quad f_{2}(t)=\tilde{f}(t)-f^{(0)}(t),
\end{gathered}
$$

and denoting

$$
Y:=\left(\bar{B}_{0} z_{1}(0 ; u), \tilde{f}_{0}-f_{0}^{(0)}\right)_{m}-\left(\bar{B}_{1} z_{4}(T ; u), \tilde{f}_{1}-f_{1}^{(0)}\right)_{n-m}+\int_{0}^{T}\left(\tilde{z}(t ; u), \tilde{f}(t)-f^{(0)}(t)\right)_{n} \mathrm{~d} t
$$

we obtain, in line with (2.7), the inequality 


$$
\begin{aligned}
|Y| \leq & \left\{\left(Q_{0}^{-1} \bar{B}_{0} z_{1}(0 ; u), \bar{B}_{0} z_{1}(0 ; u)\right)_{m}+\left(Q_{1}^{-1} \bar{B}_{1} z_{4}(T ; u), \bar{B}_{1} z_{4}(T ; u)\right)_{n-m}\right. \\
& \left.+\int_{0}^{T}\left(Q_{2}^{-1}(t) z(t ; u), z(t ; u)\right)_{n} \mathrm{~d} t\right\}^{\frac{1}{2}} \times\left\{\left(Q_{0}\left(\tilde{f}_{0}-f_{0}^{(0)}\right), \tilde{f}_{0}-f_{0}^{(0)}\right)_{m}\right. \\
& \left.+\left(Q_{1}\left(\tilde{f}_{1}-f_{1}^{(0)}\right), \tilde{f}_{1}-f_{1}^{(0)}\right)_{n-m}+\int_{0}^{T}\left(Q_{2}(t)\left(\tilde{f}(t)-f^{(0)}(t)\right), \tilde{f}(t)-f^{(0)}(t)\right)_{n} \mathrm{~d} t\right\}^{\frac{1}{2}} \\
\leq & \left\{\left(Q_{0}^{-1} \bar{B}_{0} z_{1}(0 ; u), \bar{B}_{0} z_{1}(0 ; u)\right)_{m}+\left(Q_{1}^{-1} \bar{B}_{1} z_{4}(T ; u), \bar{B}_{1} z_{4}(T ; u)\right)_{n-m}\right. \\
& \left.+\int_{0}^{T}\left(Q_{2}^{-1}(t) \tilde{z}(t ; u), \tilde{z}(t ; u)\right)_{n} \mathrm{~d} t\right\}^{\frac{1}{2}}:=q,
\end{aligned}
$$

where the equality is attained at

$$
\tilde{f}_{0}= \pm \frac{1}{q} Q_{0}^{-1} \bar{B}_{0} z_{1}(0 ; u)+f_{0}^{(0)}, \quad \tilde{f}_{1}=\mp \frac{1}{q} Q_{1}^{-1} \bar{B}_{1} Z_{4}(T ; u)+f_{1}^{(0)}, \quad \tilde{f}(t)= \pm \frac{1}{q} Q_{2}(t) z(t ; u)+f^{(0)}(t) .
$$

Thus,

$$
\begin{aligned}
& \inf _{c \in \mathbb{R}^{1}} \sup _{\tilde{F} \in G}\left[\left(\bar{B}_{0} z_{1}(0 ; u), \tilde{f}_{0}\right)_{m}+\int_{0}^{T}(z(t ; u), \tilde{f}(t))_{n} \mathrm{~d} t-\left(\bar{B}_{1} z_{4}(T ; u), \tilde{f}_{1}\right)_{n-m}-c\right]^{2} \\
&=\inf _{c \in R^{1}} \sup _{\tilde{F} \in G_{0}}\left[\left(\bar{B}_{0} z_{1}(0 ; u), \tilde{f}_{0}-f_{0}^{(0)}\right)_{m}-\left(\bar{B}_{1} z_{4}(T ; u), \tilde{f}_{1}-f_{1}^{(0)}\right)_{n-m}+\int_{0}^{T}\left(z(t ; u), \tilde{f}(t)-f^{(0)}(t)\right)_{n} \mathrm{~d} t\right. \\
&\left.+\left(\bar{B}_{0} z_{1}(0 ; u), f_{0}^{(0)}\right)_{m}-\left(\bar{B}_{1} z_{4}(T ; u), f_{1}^{(0)}\right)_{n-m}+\int_{0}^{T}\left(\tilde{z}(t ; u), f^{(0)}(t)\right)_{n} \mathrm{~d} t-c\right]^{2} \\
&= \inf _{c \in R^{1}|r| \leq q} \sup _{Y}\left[Y+\left(\bar{B}_{0} z_{1}(0 ; u), f_{0}^{(0)}\right)_{m}-\left(\bar{B}_{1} z_{4}(T ; u), f_{1}^{(0)}\right)_{n-m}+\int_{0}^{T}\left(z(t ; u), f^{(0)}(t)\right)_{n} \mathrm{~d} t-c\right]^{2}=q^{2} \\
&=\left(Q_{0}^{-1} \bar{B}_{0} z_{1}(0 ; u), \bar{B}_{0} z_{1}(0 ; u)\right)_{m}+\left(Q_{1}^{-1} \bar{B}_{1} z_{4}(T ; u), \bar{B}_{1} z_{4}(T ; u)\right)_{n-m}+\int_{0}^{T}\left(Q_{2}^{-1}(t) z(t ; u), z(t ; u)\right)_{n} \mathrm{~d} t
\end{aligned}
$$

at $c=\left(\bar{B}_{0} z_{1}(0 ; u), f_{0}^{(0)}\right)_{m}-\left(\bar{B}_{1} z_{4}(T ; u), f_{1}^{(0)}\right)_{n-m}+\int_{0}^{T}\left(z(t ; u), f^{(0)}(t)\right)_{n} \mathrm{~d} t$.

Calculate the second term on the right-hand side of (0.40). Applying the generalized Cauchy-Bunyakovsky inequality, we have

$$
\begin{aligned}
& \mathbb{E}\left[\int_{\alpha}^{s}\left(u_{1}(t), \tilde{\xi}(t)\right)_{l} \mathrm{~d} t+\int_{s}^{\beta}\left(u_{2}(t), \tilde{\xi}(t)\right)_{l} \mathrm{~d} t\right]^{2}=\mathbb{E}\left[\int_{\alpha}^{\beta}(u(t), \tilde{\xi}(t))_{l} \mathrm{~d} t\right]^{2} \\
& \leq \mathbb{E}\left[\int_{\alpha}^{\beta}\left(Q^{-1}(t) u(t), u(t)\right)_{l} \mathrm{~d} t \cdot \int_{\alpha}^{\beta}(Q(t) \xi(t), \xi(t))_{l} \mathrm{~d} t\right] \\
& =\int_{\alpha}^{\beta}\left(Q^{-1}(t) u(t), u(t)\right)_{l} \mathrm{~d} t \cdot \int_{\alpha}^{\beta} \mathbb{E}(Q(t) \tilde{\xi}(t), \tilde{\xi}(t))_{l} \mathrm{~d} t .
\end{aligned}
$$

Here $\mathbb{E}$ can be placed under the integral sign according to the Fubini theorem because we assume that $\tilde{\xi}(t)$ is a random process of the integrable second moment. Transform the last factor on the right-hand side of (0.42):

$$
\begin{aligned}
& \int_{\alpha}^{\beta} \mathbb{E}(Q(t) \tilde{\xi}(t), \tilde{\xi}(t))_{l} \mathrm{~d} t \\
& =\int_{\alpha}^{\beta} \mathbb{E}\left(\sum_{i=1}^{l}(Q(t) \tilde{\xi}(t))_{i} \tilde{\xi}_{i}(t)\right) \mathrm{d} t=\int_{\alpha}^{\beta} \mathbb{E}\left(\sum_{i=1}^{l} \sum_{k=1}^{l} q_{i k} \tilde{\xi}_{k}(t) \tilde{\xi}_{i}(t)\right) \mathrm{d} t \\
& =\int_{\alpha}^{\beta} \sum_{i=1}^{l} \sum_{k=1}^{l} q_{i k} \mathbb{E}\left(\tilde{\xi}_{k}(t) \tilde{\xi}_{i}(t)\right) \mathrm{d} t=\int_{\alpha}^{\beta} S p[Q(t) \tilde{R}(t, t)] \mathrm{d} t .
\end{aligned}
$$

Taking into account that $(0.25)$ holds, we see that $(0.42)$ yields 


$$
\begin{aligned}
& \sup _{\tilde{\xi} \in V} \mathbb{E}\left[\int_{\alpha}^{s}\left(u_{1}(t), \tilde{\xi}(t)\right)_{l} \mathrm{~d} t+\int_{s}^{\beta}\left(u_{2}(t), \tilde{\xi}(t)\right)_{l} \mathrm{~d} t\right]^{2} \\
& \leq \int_{\alpha}^{s}\left(Q^{-1}(t) u_{1}(t), u_{1}(t)\right)_{l} \mathrm{~d} t+\int_{s}^{\beta}\left(Q^{-1}(t) u_{2}(t), u_{2}(t)\right)_{l} \mathrm{~d} t .
\end{aligned}
$$

It is not difficult to check that here, the equality sign is attained at the element

$$
\tilde{\xi}(t)= \begin{cases}\frac{\eta Q^{-1}(t) u_{1}(t)}{\left[\int_{\alpha}^{s}\left(Q^{-1}(t) u_{1}(t), u_{1}(t)\right)_{l} \mathrm{~d} t+\int_{s}^{\beta}\left(Q^{-1}(t) u_{2}(t), u_{2}(t)\right)_{l} \mathrm{~d} t\right]^{1 / 2}}, & \alpha<t<s ; \\ \frac{\eta Q^{-1}(t) u_{2}(t)}{\left[\int_{\alpha}^{s}\left(Q^{-1}(t) u_{1}(t), u_{1}(t)\right)_{l} \mathrm{~d} t+\int_{s}^{\beta}\left(Q^{-1}(t) u_{2}(t), u_{2}(t)\right)_{l} \mathrm{~d} t\right]^{1 / 2}}, & s<t<\beta,\end{cases}
$$

where $\eta$ is a random variable such that $\mathbb{E} \eta=0$ and $\mathbb{E} \eta^{2}=1$. We conclude that statement of the lemma follows now from (0.40), (0.41), and (0.43).

\section{Representations for Minimax Mean Square Estimates of Functionals from Solutions to Two-Point Boundary Value Problems and Estimation Errors}

In this section we prove the theorem concerning general form of minimax mean square estimates. Solving optimal control problem (0.29), (0.30), we arrive at the following result.

Theorem 4.1 The minimax mean square estimate of expression $(a, \varphi(s))$ has the form

$$
\overline{\overline{(a, \varphi(s))_{n}}}=\int_{\alpha}^{s}\left(\hat{u}_{1}(t), y(t)\right)_{l} \mathrm{~d} t+\int_{s}^{\beta}\left(\hat{u}_{2}(t), y(t)\right)_{l} \mathrm{~d} t
$$

where

$$
\begin{gathered}
\hat{u}_{1}(t)=Q(t) H(t) p_{2}(t), \quad \hat{u}_{2}(t)=Q(t) H(t) p_{3}(t), \\
\hat{c}=\left(\bar{B}_{0} z_{1}(0), f_{0}^{(0)}\right)_{m}-\left(\bar{B}_{1} z_{4}(T), f_{1}^{(0)}\right)_{n-m}+\int_{0}^{T}\left(z(t), f^{(0)}(t)\right)_{n} \mathrm{~d} t, \\
z(t)= \begin{cases}z_{1}(t), & 0 \leq t \leq \alpha ; \\
z_{2}(t), & \alpha<t<s ; \\
z_{3}(t), & s<t<\beta ; \\
z_{4}(t), & \beta \leq t \leq T,\end{cases}
\end{gathered}
$$

and vector-functions $p_{i}(t)$ and $z_{i}(t), i=\overline{1,4}$, are determined from the solution to the problem

$$
\begin{aligned}
& L^{*} z_{1} t=0, \quad 0<t<\alpha, \quad \hat{B}_{0} z_{1}(0)=0, \\
& L^{*} z_{2}(t)=-H^{\mathrm{T}}(t) Q(t) H(t) p_{2}(t), \quad \alpha<t<s, \quad z_{2}(\alpha)=z_{1}(\alpha), \\
& L^{*} z_{3}(t)=-H^{\mathrm{T}}(t) Q(t) H(t) p_{3}(t), \quad s<t<\beta, \quad z_{3}(s)=z_{2}(s)-a, \\
& L^{*} z_{4}(t)=0, \quad \beta<t<T, \quad z_{4}(\beta)=z_{3}(\beta), \quad \hat{B}_{1} z_{4}(T)=0, \\
& L p_{1}(t)=Q_{2}^{-1}(t) z_{1}(t), \quad 0<t<\alpha, \quad B_{0} p_{1}(0)=Q_{0}^{-1} \bar{B}_{0} z_{1}(0), \\
& L p_{2}(t)=Q_{2}^{-1}(t) z_{2}(t), \quad \alpha<t<s, \quad p_{2}(\alpha)=p_{1}(\alpha), \\
& L p_{3}(t)=Q_{2}^{-1}(t) z_{3}(t), \quad s<t<\beta, \quad p_{3}(s)=p_{2}(s), \\
& L p_{4}(t)=Q_{2}^{-1}(t) z_{4}(t), \quad \beta<t<T, \quad p_{4}(\beta)=p_{3}(\beta), \\
& B_{1} p_{4}(T)=-Q_{1}^{-1} \bar{B}_{1} z_{4}(T) .
\end{aligned}
$$

Here $z_{1}, p_{1} \in\left(H^{1}(0, \alpha)\right)^{n}, \quad z_{2}, p_{2} \in\left(H^{1}(\alpha, s)\right)^{n}, \quad z_{3}, p_{3} \in\left(H^{1}(s, \beta)\right)^{n}, \quad$ and $\quad z_{4}, p_{4} \in\left(H^{1}(\beta, T)\right)^{n}$. The minimax estimation error

$$
\sigma=\left(a, p_{2}(s)\right)_{n}^{1 / 2} .
$$


Problem (0.46) is uniquely solvable.

Proof. We will solve optimal control problem (0.29), (0.30). Represent solutions $z_{i}(x ; u), i=\overline{1,4}$, of problem_(0.29) as $\bar{z}_{i}(t ; u)=\bar{z}_{i}(t ; u)+\overline{\bar{z}}_{i}(t)$, where $\quad \bar{z}_{1}(t ; u), \quad \bar{z}_{2}(t ; u), \quad \bar{z}_{3}(t ; u), \quad \bar{z}_{4}(t ; u)$ and $\overline{\bar{z}}_{1}(t)$, $\overline{\bar{Z}}_{2}(t), \quad \overline{\bar{z}}_{3}(t), \quad \overline{\bar{z}}_{4}(t)$ denote the solutions to this problem at $a=0$ and $u_{1} \equiv 0, \quad u_{2} \equiv 0$, respectively. Then function $(0.30)$ can be represented in the form

$$
I(u)=\tilde{I}(u)+2 L(u)+A,
$$

where

$$
\begin{aligned}
& \tilde{I}(u)=\left(Q_{0}^{-1} \bar{B}_{0} \bar{Z}_{1}(0 ; u), \bar{B}_{0} \bar{Z}_{1}(0 ; u)\right)_{m}+\left(Q_{1}^{-1} \bar{B}_{1} \bar{Z}_{4}(T ; u), \bar{B}_{1} \bar{Z}_{4}(T ; u)\right)_{n-m} \\
& +\int_{0}^{\alpha}\left(Q_{2}^{-1}(t) \bar{z}_{1}(t ; u), \bar{z}_{1}(t ; u)\right)_{n} \mathrm{~d} t+\int_{\alpha}^{s}\left(Q_{2}^{-1}(t) \bar{z}_{2}(t ; u), \bar{z}_{2}(t ; u)\right)_{n} \mathrm{~d} t \\
& +\int_{s}^{\beta}\left(Q_{2}^{-1}(t) \bar{Z}_{3}(t ; u), \bar{z}_{3}(t ; u)\right)_{n} \mathrm{~d} t+\int_{\beta}^{T}\left(Q_{2}^{-1}(t) \bar{Z}_{4}(t ; u), \bar{z}_{4}(t ; u)\right)_{n} \mathrm{~d} t \\
& +\int_{\alpha}^{s}\left(Q^{-1}(t) u_{1}(t), u_{1}(t)\right)_{l} \mathrm{~d} t+\int_{s}^{\beta}\left(Q^{-1}(t) u_{2}(t), u_{2}(t)\right)_{l} \mathrm{~d} t, \\
& L(u)=\left(Q_{0}^{-1} \bar{B}_{0} \bar{Z}_{1}(0 ; u), \bar{B}_{0} \overline{\bar{Z}}_{1}(0)\right)_{m}+\left(Q_{1}^{-1} \bar{B}_{1} \bar{Z}_{4}(T ; u), \bar{B}_{1} \overline{\bar{Z}}_{4}(T)\right)_{n-m} \\
& +\int_{0}^{\alpha}\left(Q_{2}^{-1}(t) \bar{z}_{1}(t ; u), \overline{\bar{z}}_{1}(t)\right)_{n} \mathrm{~d} t+\int_{\alpha}^{s}\left(Q_{2}^{-1}(t) \bar{z}_{2}(t ; u), \overline{\bar{z}}_{2}(t)\right)_{n} \mathrm{~d} t \\
& +\int_{s}^{\beta}\left(Q_{2}^{-1}(t) \bar{z}_{3}(t ; u), \overline{\bar{z}}_{3}(t)\right)_{n} \mathrm{~d} t+\int_{\beta}^{T}\left(Q_{2}^{-1}(t) \bar{z}_{4}(t ; u), \overline{\bar{z}}_{4}(t)\right)_{n} \mathrm{~d} t, \\
& A=\left(Q_{0}^{-1} \bar{B}_{0} \overline{\bar{z}}_{1}(0), \bar{B}_{0} \overline{\bar{Z}}_{1}(0)\right)_{m}+\left(Q_{1}^{-1} \bar{B}_{1} \overline{\bar{Z}}_{4}(T), \bar{B}_{1} \overline{\bar{Z}}_{4}(T)\right)_{n-m} \\
& +\int_{0}^{\alpha}\left(Q_{2}^{-1}(t) \overline{\bar{z}}_{1}(t), \overline{\bar{z}}_{1}(t)\right)_{n} \mathrm{~d} t+\int_{\alpha}^{s}\left(Q_{2}^{-1}(t) \overline{\bar{z}}_{2}(t), \overline{\bar{z}}_{2}(t)\right)_{n} \mathrm{~d} t \\
& +\int_{s}^{\beta}\left(Q_{2}^{-1}(t) \overline{\bar{z}}_{3}(t), \overline{\bar{z}}_{3}(t)\right)_{n} \mathrm{~d} t+\int_{\beta}^{T}\left(Q_{2}^{-1}(t) \overline{\bar{z}}_{4}(t), \overline{\bar{z}}_{4}(t)\right)_{n} \mathrm{~d} t .
\end{aligned}
$$

Since solution $\bar{Z}(t ; u)$ of BVP (0.31) is continuous ${ }^{2}$ with respect to right-hand side $g(t ; u)$ defined by (0.32), the function $u \rightarrow \bar{z}(\cdot ; u)$ is a linear bounded operator mapping the space $H=\left(L^{2}(\alpha, s)\right)^{l} \times\left(L^{2}(s, \beta)\right)^{l}$ to

$$
H_{1}:=\left(H^{1}(0, \alpha)\right)^{n} \times\left(H^{1}(\alpha, s)\right)^{n} \times\left(H^{1}(s, \beta)\right)^{n} \times\left(H^{1}(\beta, T)\right)^{n} .
$$

Thus, $\tilde{I}(u)$ is a continuous quadratic form corresponding to a symmetric continuous bilinear form

$$
\begin{aligned}
\pi(u, v):= & \left(Q_{0}^{-1} \bar{B}_{0} \bar{z}_{1}(0 ; u), \bar{B}_{0} \bar{z}_{1}(0 ; v)\right)_{m}+\left(Q_{1}^{-1} \bar{B}_{1} \bar{z}_{4}(T ; u), \bar{B}_{1} \bar{z}_{4}(T ; v)\right)_{n-m} \\
& +\int_{0}^{\alpha}\left(Q_{2}^{-1}(t) \bar{z}_{1}(t ; u), \bar{z}_{1}(t ; v)\right)_{n} \mathrm{~d} t+\int_{\alpha}^{s}\left(Q_{2}^{-1}(t) \bar{z}_{2}(t ; u), \bar{z}_{2}(t ; v)\right)_{n} \mathrm{~d} t \\
& +\int_{s}^{\beta}\left(Q_{2}^{-1}(t) \bar{z}_{3}(t ; u), \bar{z}_{3}(t ; v)\right)_{n} \mathrm{~d} t+\int_{\beta}^{T}\left(Q_{2}^{-1}(t) \bar{z}_{4}(t ; u), \bar{z}_{4}(t ; v)\right)_{n} \mathrm{~d} t \\
& +\int_{\alpha}^{s}\left(Q^{-1}(t) u_{1}(t), v_{1}(t)\right)_{1} \mathrm{~d} t+\int_{s}^{\beta}\left(Q^{-1}(t) u_{2}(t), v_{2}(t)\right)_{l} \mathrm{~d} t,
\end{aligned}
$$

$L(u)$ is a linear continuous functional defined on $H$, and $A$ is a constant independent of $u$. We have

$$
\begin{aligned}
\tilde{I}(u) & =\tilde{I}\left(u_{1}, u_{2}\right) \geq \int_{\alpha}^{s}\left(Q^{-1}(t) u_{1}(t), u_{1}(t)\right)_{l} \mathrm{~d} t+\int_{s}^{\beta}\left(Q^{-1}(t) u_{2}(t), u_{2}(t)\right)_{l} \mathrm{~d} t \\
& \geq c\|u\|_{H}^{2}, \quad c=\text { const. }
\end{aligned}
$$

Using Theorem 1.1 from [17], we conclude that there is one and only one element $\hat{u}=\left(\hat{u}_{1}, \hat{u}_{2}\right) \in H$ such that

$$
I(\hat{u})=I\left(\hat{u}_{1}, \hat{u}_{2}\right)=\inf _{\left(u_{1}, u_{2}\right) \in H} I\left(u_{1}, u_{2}\right) .
$$

${ }^{2}$ This continuous dependence follows from the representation of function $\bar{z}(t ; u)$ in terms of Green's matrix $G^{*}(t, \xi)$ of BVP $(0.31)$ (see [13], p. 115):

$$
\bar{z}(t ; u)=-\int_{\alpha}^{s} G^{*}(t, \xi) H^{\mathrm{T}}(\xi) u_{1}(\xi) \mathrm{d} \xi-\int_{s}^{\beta} G^{*}(t, \xi) H^{\mathrm{T}}(\xi) u_{2}(\xi) \mathrm{d} \xi .
$$


Therefore

$$
\left.\frac{\mathrm{d}}{\mathrm{d} \tau} I\left(\hat{u}_{1}+\tau v_{1}, \hat{u}_{2}+\tau v_{2}\right)\right|_{\tau=0}=0 \quad \forall v=\left(v_{1}, v_{2}\right) \in H .
$$

Taking into consideration the latter equality, (0.30), and designations on p. 11, we obtain

$$
\begin{aligned}
0= & \left.\frac{1}{2} \frac{\mathrm{d} I(\hat{u}+\tau v)}{\mathrm{d} \tau}\right|_{\tau=0}=\int_{\alpha}^{s}\left(Q^{-1}(t) \hat{u}_{1}, v_{1}\right)_{l} \mathrm{~d} t+\int_{s}^{\beta}\left(Q^{-1}(t) \hat{u}_{2}, v_{2}\right)_{l} \mathrm{~d} t \\
& +\left(Q_{0}^{-1} \bar{B}_{0} z_{1}(0 ; \hat{u}), \bar{B}_{0} \bar{z}_{1}(0 ; v)\right)_{m}+\left(Q_{1}^{-1} \bar{B}_{1} z_{4}(T ; \hat{u}), \bar{B}_{1} \bar{z}_{4}(T ; v)\right)_{n-m} \\
& +\int_{0}^{\alpha}\left(Q_{2}^{-1}(t) z_{1}(t ; \hat{u}), \bar{z}_{1}(t ; v)\right)_{n} \mathrm{~d} t+\int_{\alpha}^{s}\left(Q_{2}^{-1}(t) z_{2}(t ; \hat{u}), \bar{z}_{2}(t ; v)\right)_{n} \mathrm{~d} t \\
& +\int_{s}^{\beta}\left(Q_{2}^{-1}(t) z_{3}(t ; \hat{u}), \bar{z}_{3}(t ; v)\right)_{n} \mathrm{~d} t+\int_{\beta}^{T}\left(Q_{2}^{-1}(t) z_{4}(t ; \hat{u}), \bar{z}_{4}(t ; v)\right)_{n} \mathrm{~d} t .
\end{aligned}
$$

Introduce functions $p_{1} \in\left(H^{1}(0, \alpha)\right)^{n}, \quad p_{2} \in\left(H^{1}(\alpha, s)\right)^{n}, \quad p_{3} \in\left(H^{1}(s, \beta)\right)^{n}$, and $p_{4} \in\left(H^{1}(\beta, T)\right)^{n}$ as a unique solution to the following problem ${ }^{3}$ :

$$
\begin{aligned}
& L p_{1}(t)=Q_{2}^{-1}(t) z_{1}(t ; \hat{u}), \quad 0<t<\alpha, \quad B_{0} p_{1}(0)=Q_{0}^{-1} \bar{B}_{0} z_{1}(0 ; \hat{u}), \\
& L p_{2}(t)=Q_{2}^{-1}(t) z_{2}(t ; \hat{u}), \quad \alpha<t<s, \quad p_{2}(\alpha)=p_{1}(\alpha), \\
& L p_{3}(t)=Q_{2}^{-1}(t) z_{3}(t ; \hat{u}), \quad s<t<\beta, \quad p_{3}(s)=p_{2}(s), \\
& L p_{4}(t)=Q_{2}^{-1}(t) z_{4}(t ; \hat{u}), \quad \beta<t<T, \\
& p_{4}(\beta)=p_{3}(\beta), \quad B_{1} p_{4}(T)=-Q_{1}^{-1} \bar{B}_{1} z_{4}(T ; \hat{u}) .
\end{aligned}
$$

Now transform the sum of the last for terms on the right-hand side of $(0.48)$ taking into notice that $\left(\bar{Z}_{1}(0 ; v), p_{1}(0)\right)_{n}=\left(\bar{B}_{0} \bar{z}_{1}(0 ; v), B_{0} p_{1}(0)\right)_{m}$ and $\left(\bar{Z}_{4}(T ; v), p_{4}(T)\right)_{n}=\left(\bar{B}_{1} \bar{Z}_{4}(T ; v), B_{1} p_{4}(T)\right)_{n-m}$. We have

$$
\begin{aligned}
\int_{0}^{\alpha} & \left(Q_{2}^{-1}(t) z_{1}(t ; \hat{u}), \bar{z}_{1}(t ; v)\right)_{n} \mathrm{~d} t+\int_{\alpha}^{s}\left(Q_{2}^{-1}(t) z_{2}(t ; \hat{u}), \bar{z}_{2}(t ; v)\right)_{n} \mathrm{~d} t \\
+ & \int_{s}^{\beta}\left(Q_{2}^{-1}(t) z_{3}(t ; \hat{u}), \bar{z}_{3}(t ; v)\right)_{n} \mathrm{~d} t+\int_{\beta}^{T}\left(Q_{2}^{-1}(t) z_{4}(t ; \hat{u}), \bar{z}_{4}(t ; v)\right)_{n} \mathrm{~d} t \\
= & \int_{0}^{\alpha}\left(L p_{1}(t), \bar{z}_{1}(t ; v)\right)_{n} \mathrm{~d} t+\int_{\alpha}^{s}\left(L p_{2}(t), \bar{z}_{2}(t ; v)\right)_{n} \mathrm{~d} t \\
& +\int_{s}^{\beta}\left(L p_{3}(t), \bar{z}_{3}(t ; v)\right)_{n} \mathrm{~d} t+\int_{\beta}^{T}\left(L p_{4}(t), \bar{z}_{4}(t ; v)\right)_{n} \mathrm{~d} t \\
= & \int_{0}^{\alpha}\left(p_{1}(t), L^{*} \bar{z}_{1}(t ; v)\right)_{n} \mathrm{~d} t+\left(\bar{z}_{1}(\alpha ; v), p_{1}(\alpha)\right)_{n}-\left(\bar{z}_{1}(0 ; v), p_{1}(0)\right)_{n} \\
& +\int_{\alpha}^{s}\left(p_{2}(t), L^{*} \bar{z}_{2}(t ; v)\right)_{n} \mathrm{~d} t+\left(\bar{z}_{2}(s ; v), p_{2}(s)\right)_{n}-\left(\bar{z}_{2}(\alpha ; v), p_{2}(\alpha)\right)_{n} \\
& +\int_{s}^{\beta}\left(p_{3}(t), L^{*} \bar{z}_{3}(t ; v)\right)_{n} \mathrm{~d} t+\left(\bar{z}_{3}(\beta ; v), p_{3}(\beta)\right)_{n}-\left(\bar{z}_{3}(s ; v), p_{3}(s)\right)_{n} \\
& +\int_{\beta}^{T}\left(p_{4}(t), L^{*} \bar{z}_{4}(t ; v)\right)_{n} \mathrm{~d} t+\left(\bar{z}_{4}(T ; v), p_{4}(T)\right)_{n}-\left(\bar{z}_{4}(\beta ; v), p_{4}(\beta)\right)_{n} \\
= & -\left(\bar{z}_{1}(0 ; v), p_{1}(0)\right)_{n}+\left(\bar{z}_{4}(T ; v), p_{4}(T)\right)_{n}-\int_{\alpha}^{s}\left(p_{2}(t), H^{T}(t) v_{1}(t)\right)_{n} \mathrm{~d} t \\
& -\int_{s}^{\beta}\left(p_{3}(t), H^{\mathrm{T}}(t) v_{2}(t)\right)_{n} \mathrm{~d} t+\left(\bar{z}_{2}(s ; v)-\bar{z}_{3}(s ; v), p_{2}(s)\right)_{n} \\
= & -\left(\bar{B}_{0} \bar{z}_{1}(0 ; v), B_{0} p_{1}(0)\right)_{m}+\left(\bar{B}_{1} \bar{z}_{4}(T ; v), B_{1} p_{4}(T)\right)_{n-m} \\
& -\int_{\alpha}^{s}\left(H(t) p_{2}(t), v_{1}(t)\right)_{l} \mathrm{~d} t-\int_{s}^{\beta}\left(H(t) p_{3}(t), v_{2}(t)\right)_{l} \mathrm{~d} t \\
= & -\left(\bar{B}_{0} \bar{z}_{1}(0 ; v), Q_{0}^{-1} \bar{B}_{0} z_{1}(0 ; \hat{u})\right)_{m}-\left(B_{1} \bar{z}_{4}(T ; v), Q_{1}^{-1} \bar{B}_{1} z_{4}(T ; \hat{u})\right)_{n-m} \\
& -\int_{\alpha}^{s}\left(H(t) p_{2}(t), v_{1}(t)\right)_{l} \mathrm{~d} t-\int_{s}^{\beta}\left(H(t) p_{3}(t), v_{2}(t)\right)_{l} \mathrm{~d} t \\
&
\end{aligned}
$$

\footnotetext{
${ }^{3}$ The unique solvability of problem (0.49) can be proved similarly to problem (0.29).
} 
From Equalities (0.48)-(0.50) it follows that

$$
\begin{aligned}
& \int_{\alpha}^{s}\left(Q^{-1}(t) \hat{u}_{1}(t), v_{1}(t)\right)_{l} \mathrm{~d} t+\int_{s}^{\beta}\left(Q^{-1}(t) \hat{u}_{2}(t), v_{2}(t)\right)_{l} \mathrm{~d} t \\
& =\int_{\alpha}^{s}\left(H(t) p_{2}(t), v_{1}(t)\right)_{l} \mathrm{~d} t+\int_{s}^{\beta}\left(H(t) p_{3}(t), v_{2}(t)\right)_{l} \mathrm{~d} t,
\end{aligned}
$$

so that

$$
\begin{aligned}
& Q^{-1}(t) \hat{u}_{1}(t)=H(t) p_{2}(t), \quad Q^{-1}(t) \hat{u}_{2}(t)=H(t) p_{3}(t), \\
& \hat{u}_{1}(t)=Q(t) H(t) p_{2}(t), \quad \hat{u}_{2}(t)=Q(t) H(t) p_{3}(t) .
\end{aligned}
$$

Functions $p_{1}(t), \quad p_{2}(t), \quad p_{3}(t)$, and $p_{4}(t)$ are absolutely continuous on segments $[0, \alpha],[\alpha, s]$, $[s, \beta]$, and $[\beta, T]$, respectively, as solutions to BVP $(0.49)$; therefore, functions $\hat{u}_{1}(t)$ and $\hat{u}_{2}(t)$ that perform optimal control are continuous on $[\alpha, s]$ and $[s, \beta]$. Replacing in (0.29) functions $u_{1}(t)$ and $u_{2}(t)$ by $\hat{u}_{1}(t)$ and $\hat{u}_{2}(t)$ defined by formulas $(0.51)$ and denoting $z_{i}(t)=z_{i}(t ; \hat{u}), \quad i=\overline{1,4}$, we arrive at problem (0.46) and equalities (0.44).

Taking into consideration the way this problem was formulated we can state that its unique solvability follows from the fact that functional (0.30) has one minimum point $\hat{u}$.

Now let us prove representation (0.47). Substituting into formula $\sigma^{2}=I(\hat{u})$ expressions $(0.44)$ for $\hat{u}_{1}(t)$ and $\hat{u}_{2}(t)$, we have

$$
\begin{aligned}
\sigma^{2}= & \left(Q_{0}^{-1} \bar{B}_{0} z_{1}(0), \bar{B}_{0} z_{1}(0)\right)_{m}+\left(Q_{1}^{-1} \bar{B}_{1} z_{4}(T), \bar{B}_{1} z_{4}(T)\right)_{n-m} \\
& +\int_{0}^{\alpha}\left(Q_{2}^{-1}(t) z_{1}(t), z_{1}(t)\right)_{n} \mathrm{~d} t+\int_{\alpha}^{s}\left(Q_{2}^{-1}(t) z_{2}(t), z_{2}(t)\right)_{n} \mathrm{~d} t \\
& +\int_{s}^{\beta}\left(Q_{2}^{-1}(t) z_{3}(t), z_{3}(t)\right)_{n} \mathrm{~d} t+\int_{\beta}^{T}\left(Q_{2}^{-1}(t) z_{4}(t), z_{4}(t)\right)_{n} \mathrm{~d} t \\
& +\int_{\alpha}^{s}\left(Q(t) H(t) p_{2}(t), H(t) p_{2}(t)\right)_{l} \mathrm{~d} t \\
& +\int_{s}^{\beta}\left(Q(t) H(t) p_{3}(t), H(t) p_{3}(t)\right)_{l} \mathrm{~d} t .
\end{aligned}
$$

Next, we can apply the reasoning similar to that on p. 4 and use (0.46) to obtain

$$
\left(z_{1}(0), p_{1}(0)\right)_{n}=\left(\bar{B}_{0} Z_{1}(0), B_{0} p_{1}(0)\right)_{m}=\left(\bar{B}_{0} Z_{1}(0), Q_{0}^{-1} \bar{B}_{0} Z_{1}(0)\right)_{m},
$$

which yields

$$
\begin{aligned}
& \int_{0}^{\alpha}\left(Q_{2}^{-1}(t) z_{1}(t), z_{1}(t)\right)_{n} \mathrm{~d} t+\int_{\alpha}^{s}\left(Q(t) H(t) p_{2}(t), H(t) p_{2}(t)\right)_{l} \mathrm{~d} t \\
& =\int_{0}^{\alpha}\left(L p_{1}(t), z_{1}(t)\right)_{n} \mathrm{~d} t-\int_{\alpha}^{s}\left(L^{*} z_{2}(t), p_{2}(t)\right)_{n} \mathrm{~d} t \\
& =\int_{0}^{\alpha}\left(p_{1}(t), L^{*} z_{1}(t)\right)_{n} \mathrm{~d} t+\left(z_{1}(\alpha), p_{1}(\alpha)\right)_{n}-\left(z_{1}(0), p_{1}(0)\right)_{n} \\
& \quad-\int_{\alpha}^{s}\left(z_{2}(t), L p_{2}(t)\right)_{n} \mathrm{~d} t-\left(z_{2}(\alpha), p_{2}(\alpha)\right)_{n}+\left(z_{2}(s), p_{2}(s)\right)_{n} \\
& =-\left(z_{1}(0), p_{1}(0)\right)_{n}-\int_{\alpha}^{s}\left(z_{2}(t), Q_{2}^{-1}(t) z_{2}(t)\right)_{n} \mathrm{~d} t+\left(z_{2}(s), p_{2}(s)\right)_{n} \\
& =-\int_{\alpha}^{s}\left(Q_{2}^{-1}(t) z_{2}(t), z_{2}(t)\right)_{n} \mathrm{~d} t-\left(\bar{B}_{0} z_{1}(0), Q_{0}^{-1} \bar{B}_{0} z_{1}(0)\right)_{m}+\left(z_{2}(s), p_{2}(s)\right)_{n} .
\end{aligned}
$$

In a similar manner, using the equality

$$
\left(z_{4}(T), p_{4}(T)\right)_{n}=\left(\bar{B}_{1} Z_{4}(T), B_{1} p_{4}(T)\right)_{n-m}=-\left(\bar{B}_{1} Z_{4}(T), Q_{1}^{-1} \bar{B}_{1} Z_{4}(T)\right)_{n-m},
$$

we obtain 


$$
\begin{aligned}
\int_{\beta}^{T}\left(Q_{2}^{-1}(t) z_{4}(t), z_{4}(t)\right)_{n} \mathrm{~d} t+\int_{s}^{\beta}\left(Q(t) H(t) p_{3}(t), H(t) p_{3}(t)\right)_{l} \mathrm{~d} t \\
=\int_{\beta}^{T}\left(L p_{4}(t), z_{4}(t)\right)_{n} \mathrm{~d} t-\int_{s}^{\beta}\left(L^{*} z_{3}(t), p_{3}(t)\right)_{n} \mathrm{~d} t \\
=\int_{\beta}^{T}\left(p_{4}(t), L^{*} z_{4}(t)\right)_{n} \mathrm{~d} t+\left(p_{4}(T), z_{4}(T)\right)_{n}-\left(p_{4}(\beta), z_{4}(\beta)\right)_{n} \\
\quad-\int_{s}^{\beta}\left(z_{3}(t), L p_{3}(t)\right)_{n} \mathrm{~d} t+\left(z_{3}(\beta), p_{3}(\beta)\right)_{n}-\left(z_{3}(s), p_{3}(s)\right)_{n} \\
=\left(p_{4}(T), z_{4}(T)\right)_{n}-\int_{s}^{\beta}\left(z_{3}(t), Q_{2}^{-1}(t) z_{3}(t)\right)_{n} \mathrm{~d} t-\left(z_{3}(s), p_{2}(s)\right)_{n} \\
=-\int_{s}^{\beta}\left(Q_{2}^{-1}(t) z_{3}(t), z_{3}(t)\right)_{n} \mathrm{~d} t-\left(\bar{B}_{1} z_{4}(T), Q_{1}^{-1} \bar{B}_{1} z_{4}(T)\right)_{n-m}-\left(z_{3}(s), p_{2}(s)\right)_{n} .
\end{aligned}
$$

Relationships (0.52)-(0.54) yield

$$
\sigma^{2}=\left(a, p_{2}(s)\right)_{n},
$$

which is to be proved.

It is easy to see that function $z(t)$ defined by (0.45) and the function

$$
p(t)= \begin{cases}p_{1}(t), & 0 \leq t \leq \alpha ; \\ p_{2}(t), & \alpha<t \leq s ; \\ p_{3}(t), & s<t \leq \beta ; \\ p_{4}(t), & \beta<t \leq T,\end{cases}
$$

satisfy the following uniquely solvable BVP

$$
\begin{aligned}
& L^{*} z(t)=\chi_{(\alpha, \beta)}(t) H^{\mathrm{T}}(t) Q(t) H(t) p_{2}(t), \quad 0<t<T, \\
& \hat{B}_{0} z(0)=0,\left.\quad[z]\right|_{t=s}=-a, \quad \hat{B}_{1} z(T)=0, \\
& L p(t)=Q_{2}^{-1}(t) z_{1}(t), \quad 0<t<T, \\
& B_{0} p(0)=Q_{0}^{-1} \bar{B}_{0} z(0), \quad B_{1} p(T)=-Q_{1}^{-1} \bar{B}_{1} z(T),
\end{aligned}
$$

where

$$
\begin{gathered}
{\left.[z]\right|_{t=s}=z(s+0)-z(s-0),} \\
p \in\left(H^{1}(0, T)\right)^{n}, \\
\left.z\right|_{(0, s)} \in\left(H^{1}(0, s)\right)^{n},\left.\quad z\right|_{(s, T)} \in\left(H^{1}(s, T)\right)^{n},
\end{gathered}
$$

and $\chi(t)$ is the characteristic function of interval $(\alpha, \beta)$.

Now Theorem 4.1 can be restated as follows:

Theorem 4.1' The minimax estimate of expression $(a, \varphi(s))_{n}$ has the form

$$
\overline{\overline{(a, \varphi(s))_{n}}}=\int_{\beta}^{\alpha}(\hat{u}(t), y(t))_{l} \mathrm{~d} t+\hat{c},
$$

where

$$
\begin{gathered}
\hat{u}(t)=Q(t) H(t) p(t), \\
\hat{c}=\left(\bar{B}_{0} z(0), f_{0}^{(0)}\right)_{m}-\left(\bar{B}_{1} z(T), f_{1}^{(0)}\right)_{n-m}+\int_{0}^{T}\left(z(t), f^{(0)}(t)\right)_{n} \mathrm{~d} t,
\end{gathered}
$$

and vector-functions $z(t)$ and $p(t)$ are determined from the solution to problem 0.56.

Obtain now another representation for the minimax mean square estimate of quantity $(a, \varphi(s))_{n}$ which is independent of $a$ and $s$. To this end, introduce vector-functions $\hat{p}_{1}, \hat{\varphi}_{1} \in\left(H^{1}(0, \alpha)\right)^{n}, \quad \hat{p}_{2}, \hat{\varphi}_{2} \in\left(H^{1}(\alpha, s)\right)^{n}$, 
$\hat{p}_{3}, \hat{\varphi}_{3} \in\left(H^{1}(s, \beta)\right)^{n}$, and $\hat{p}_{4}, \hat{\varphi}_{4} \in\left(H^{1}(\beta, T)\right)^{n}$ as solutions to the problem

$$
\begin{aligned}
& L^{*} \hat{p}_{1}(t)=0, \quad 0<t<\alpha, \quad \hat{B}_{0} \hat{p}_{1}(0)=0, \\
& L^{*} \hat{p}_{2}(t)=H^{\mathrm{T}}(t) Q(t)\left(y(t)-H(t) \hat{\varphi}_{2}(t)\right), \quad \alpha<t<s, \quad \hat{p}_{2}(\alpha)=\hat{p}_{1}(\alpha), \\
& L^{*} \hat{p}_{3}(t)=H^{\mathrm{T}}(t) Q(t)\left(y(t)-H(t) \hat{\varphi}_{3}(t)\right), \quad s<t<\beta, \quad \hat{p}_{3}(s)=\hat{p}_{2}(s), \\
& L^{*} \hat{p}_{4}(t)=0, \quad \beta<t<T, \quad \hat{p}_{4}(\beta)=\hat{p}_{3}(\beta), \quad \hat{B}_{1} \hat{p}_{4}(T)=0, \\
& L \hat{\varphi}_{1}(t)=Q_{2}^{-1}(t) \hat{p}_{1}(t)+f^{(0)}(t), \quad 0<t<\alpha, \quad B_{0} \hat{\varphi}_{1}(0)=Q_{0}^{-1} \bar{B}_{0} \hat{p}_{1}(0)+f_{0}^{(0)}, \\
& L \hat{\varphi}_{2}(t)=Q_{2}^{-1}(t) \hat{p}_{2}(t)+f^{(0)}(t), \quad \alpha<t<s, \quad \hat{\varphi}_{2}(\alpha)=\hat{\varphi}_{1}(\alpha), \\
& L \hat{\varphi}_{3}(t)=Q_{2}^{-1}(t) \hat{p}_{3}(t)+f^{(0)}(t), \quad s<t<\beta, \quad \hat{\varphi}_{3}(s)=\hat{\varphi}_{2}(s), \\
& L \hat{\varphi}_{4}(t)=Q_{2}^{-1}(t) \hat{p}_{4}(t)+f^{(0)}(t), \quad \beta<t<T, \\
& \hat{\varphi}_{4}(\beta)=\hat{\varphi}_{3}(\beta), \quad B_{1} \hat{\varphi}_{4}(T)=-Q_{1}^{-1} \bar{B}_{1} \hat{p}_{4}(T)-f_{1}^{(0)}
\end{aligned}
$$

at realizations $y$ that belong with probability 1 to space $\left(L^{2}(\alpha, \beta)\right)^{l}$.

Note that unique solvability of problem $(0.58)$ at every realization can be proved similarly to the case of (0.46). Namely, one can show that solutions to the problem of optimal control of the system

$$
\begin{gathered}
L^{*} \hat{p}_{1}(t ; v)=0, \quad 0<t<\alpha, \quad \hat{B}_{0} \hat{p}_{1}(0 ; v)=0, \\
L^{*} \hat{p}_{2}(t ; v)=d(t)-H^{\mathrm{T}}(t) v_{1}(t), \quad \alpha<t<s, \\
\hat{p}_{1}(\alpha ; v)=\hat{p}_{2}(\alpha ; v), \\
L^{*} \hat{p}_{3}(t ; v)=d(t)-H^{\mathrm{T}}(t) v_{2}(t), \quad s<t<\beta, \\
\hat{p}_{2}(s ; v)=\hat{p}_{3}(s ; v), \\
L^{*} \hat{p}_{4}(t ; v)=0, \quad \beta<t<T, \\
\hat{p}_{4}(\beta ; v)=\hat{p}_{3}(\beta ; v), \quad \hat{B}_{1} \hat{p}_{4}(T ; v)=0
\end{gathered}
$$

with the cost function

$$
\begin{aligned}
& J(v)=\left(Q_{0}^{-1}\left(\bar{B}_{0} \hat{p}_{1}(0 ; v)+Q_{0} f_{0}^{(0)}\right), \bar{B}_{0} \hat{p}_{1}(0 ; v)+Q_{0} f_{0}^{(0)}\right)_{m} \\
&+\left(Q_{1}^{-1}\left(\bar{B}_{1} \hat{p}_{4}(T ; v)+Q_{1} f_{1}^{(0)}\right), \bar{B}_{1} \hat{p}_{4}(T ; v)+Q_{1} f_{1}^{(0)}\right)_{n-m} \\
&+\int_{0}^{\alpha}\left(Q_{2}^{-1}(t)\left(\hat{p}_{1}(t ; v)+Q_{2}(t) f^{(0)}(t)\right), \hat{p}_{1}(t ; v)+Q_{2}(t) f^{(0)}(t)\right)_{n} \mathrm{~d} t \\
&+\int_{\alpha}^{s}\left(Q_{2}^{-1}(t)\left(\hat{p}_{2}(t ; v)+Q_{2}(t) f^{(0)}(t)\right), \hat{p}_{2}(t ; v)+Q_{2}(t) f^{(0)}(t)\right)_{n} \mathrm{~d} t \\
&+\int_{s}^{\beta}\left(Q_{2}^{-1}(t)\left(\hat{p}_{3}(t ; v)+Q_{2}(t) f^{(0)}(t)\right), \hat{p}_{3}(t ; v)+Q_{2}(t) f^{(0)}(t)\right)_{n} \mathrm{~d} t \\
&+\int_{\beta}^{T}\left(Q_{2}^{-1}(t)\left(\hat{p}_{4}(t ; v)+Q_{2}(t) f^{(0)}(t)\right), \hat{p}_{4}(t ; v)+Q_{2}(t) f^{(0)}(t)\right)_{n} \mathrm{~d} t \\
&+\int_{\alpha}^{s}\left(Q^{-1}(t) v_{1}(t), v_{1}(t)\right)_{l} \mathrm{~d} t+\int_{s}^{\beta}\left(Q^{-1}(t) v_{2}(t), v_{2}(t)\right)_{l} \mathrm{~d} t \\
& \rightarrow \min _{v=\left(v_{1}, v_{2}\right) \in H^{\prime}} \quad d(t)=H^{\mathrm{T}}(t) Q(t) y(t), \alpha<t<\beta,
\end{aligned}
$$

can be reduced to the solution of problem (0.58) where the optimal control $\hat{v}=\left(\hat{v}_{1}, \hat{v}_{2}\right)$ is expressed in terms of the solution to this problem as $\hat{v}_{1}=Q(t) H(t) \hat{\varphi}_{2}(t), \hat{v}_{2}=Q(t) H(t) \hat{\varphi}_{3}(t)$; the unique solvability of the problem follows from the existence of the unique minimum point $\hat{v}$ of functional $J(v)$. 
Considering system (0.58) at realizations $y$ it is easy to see that its solution is continuous with respect to the right-hand side. This property enables us to conclude, using the general theory of linear continuous transformations of random processes, that the functions $\hat{p}_{i}(t), \quad \hat{\varphi}_{1}(t), \quad i=\overline{1,4}$, considered as random fields have finite second moments.

Theorem 4.2 The following representation is valid

$$
\overline{\overline{(a, \varphi(s))_{n}}}=\left(a, \hat{\varphi}_{2}(s)\right)_{n} \text {. }
$$

Proof. By virtue of (0.44) and (0.58),

$$
\begin{aligned}
\overline{\overline{(a, \varphi(s))_{n}}} & =\int_{\alpha}^{s}\left(\hat{u}_{1}(t), y(t)\right)_{l} \mathrm{~d} t+\int_{s}^{\beta}\left(\hat{u}_{2}(t), y(t)\right)_{l} \mathrm{~d} t+\hat{c} \\
& =\int_{\alpha}^{s}\left(Q(t) H(t) p_{2}(t), y(t)\right)_{l} \mathrm{~d} t+\int_{\alpha}^{s}\left(Q(t) H(t) p_{3}(t), y(t)\right)_{l} \mathrm{~d} t+\hat{c} \\
& =\int_{\alpha}^{s}\left(p_{2}(t), H^{\mathrm{T}}(t) Q(t) y(t)\right)_{n} \mathrm{~d} t+\int_{\alpha}^{s}\left(p_{3}(t), H^{\mathrm{T}}(t) Q(t) y(t)\right)_{n} \mathrm{~d} t+\hat{c} .
\end{aligned}
$$

Next,

$$
\begin{aligned}
\int_{\alpha}^{s}\left(p_{2}(t), H^{\mathrm{T}}(t) Q(t) y(t)\right)_{l} \mathrm{~d} t \\
=\int_{\alpha}^{s}\left(p_{2}(t), L^{*} \hat{p}_{2}(t)\right)_{n} \mathrm{~d} t+\int_{\alpha}^{s}\left(p_{2}(t), H^{\mathrm{T}}(t) Q(t) H(t) \hat{\varphi}_{2}(t)\right)_{n} \mathrm{~d} t \\
=\int_{\alpha}^{s}\left(L p_{2}(t), \hat{p}_{2}(t)\right)_{n} \mathrm{~d} t+\left(p_{2}(\alpha), \hat{p}_{2}(\alpha)\right)_{n}-\left(p_{2}(s), \hat{p}_{2}(s)\right)_{n} \\
\quad+\int_{\alpha}^{s}\left(H^{\mathrm{T}}(t) Q(t) H(t) p_{2}(t), \hat{\varphi}_{2}(t)\right)_{n} \mathrm{~d} t \\
=\int_{\alpha}^{s}\left(Q_{2}^{-1}(t) z_{2}(t), \hat{p}_{2}(t)\right)_{n} \mathrm{~d} t+\left(p_{2}(\alpha), \hat{p}_{2}(\alpha)\right)_{n} \\
\quad-\left(p_{2}(s), \hat{p}_{2}(s)\right)_{n}-\int_{\alpha}^{s}\left(L^{*} z_{2}(t), \hat{\varphi}_{2}(t)\right)_{n} \mathrm{~d} t \\
=\int_{\alpha}^{s}\left(z_{2}(t), L \hat{\varphi}_{2}(t)\right)_{n} \mathrm{~d} t+\left(p_{2}(\alpha), \hat{p}_{2}(\alpha)\right)_{n}-\left(p_{2}(s), \hat{p}_{2}(s)\right)_{n} \\
\quad-\int_{\alpha}^{s}\left(z_{2}(t), L \hat{\varphi}_{2}(t)\right)_{n} \mathrm{~d} t+\left(z_{2}(s), \hat{\varphi}_{2}(s)\right)_{n} \\
\quad-\left(z_{2}(\alpha), \hat{\varphi}_{2}(\alpha)\right)_{n}-\int_{\alpha}^{s}\left(z_{2}(t), f^{(0)}(t)\right)_{n} \mathrm{~d} t \\
=\left(p_{2}(\alpha), \hat{p}_{2}(\alpha)\right)_{n}-\left(p_{2}(s), \hat{p}_{2}(s)\right)_{n}+\left(z_{2}(s), \hat{\varphi}_{2}(s)\right)_{n} \\
\quad-\left(z_{2}(\alpha), \hat{\varphi}_{2}(\alpha)\right)_{n}-\int_{\alpha}^{s}\left(z_{2}(t), f^{(0)}(t)\right)_{n} \mathrm{~d} t .
\end{aligned}
$$

Similarly,

$$
\begin{aligned}
& \int_{s}^{\beta}\left(p_{3}(t), H^{\mathrm{T}}(t) Q(t) y(t)\right)_{l} \mathrm{~d} t \\
& =\left(p_{3}(s), \hat{p}_{3}(s)\right)_{n}-\left(p_{3}(\beta), \hat{p}_{3}(\beta)\right)_{n}+\left(z_{3}(\beta), \hat{\varphi}_{3}(\beta)\right)_{n} \\
& \quad-\left(z_{3}(s), \hat{\varphi}_{3}(s)\right)_{n}-\int_{s}^{\beta}\left(z_{3}(t), f^{(0)}(t)\right)_{n} \mathrm{~d} t
\end{aligned}
$$

From (0.59), (0.60), and (0.61) it follows

$$
\begin{aligned}
\overline{\overline{(a, \varphi(s))}=} & \left(p_{1}(\alpha), \hat{p}_{1}(\alpha)\right)_{n}-\int_{\alpha}^{s}\left(z_{2}(t), f^{(0)}(t)\right)_{n} \mathrm{~d} t-\int_{s}^{\beta}\left(z_{3}(t), f^{(0)}(t)\right)_{n} \mathrm{~d} t \\
& -\left(z_{1}(\alpha), \hat{\varphi}_{1}(\alpha)\right)_{n}-\left(p_{4}(\beta), \hat{p}_{4}(\beta)\right)_{n}+\left(z_{4}(\beta), \hat{\varphi}_{4}(\beta)\right)_{n}+\left(a, \hat{\varphi}_{2}(s)\right)_{n}+\hat{c} .
\end{aligned}
$$

However,

$$
\begin{aligned}
0 & =\int_{0}^{\alpha}\left(\hat{\varphi}_{1}(t), L^{*} z_{1}(t)\right)_{n} \mathrm{~d} t=\int_{0}^{\alpha}\left(L \hat{\varphi}_{1}(t), z_{1}(t)\right)_{n} \mathrm{~d} t-\left(z_{1}(\alpha), \hat{\varphi}_{1}(\alpha)\right)_{n}+\left(z_{1}(0), \hat{\varphi}_{1}(0)\right)_{n} \\
& =\int_{0}^{\alpha}\left(z_{1}(t), Q_{2}(t) \hat{p}_{1}(t)\right)_{n} \mathrm{~d} t+\int_{0}^{\alpha}\left(z_{1}(t), f^{(0)}(t)\right)_{n} \mathrm{~d} t-\left(z_{1}(\alpha), \hat{\varphi}_{1}(\alpha)\right)_{n}+\left(z_{1}(0), \hat{\varphi}_{1}(0)\right)_{n},
\end{aligned}
$$




$$
\begin{aligned}
0 & =\int_{0}^{\alpha}\left(L^{*} \hat{p}_{1}(t), p_{1}(t)\right)_{n} \mathrm{~d} t=\int_{0}^{\alpha}\left(\hat{p}_{1}(t), L p_{1}(t)\right)_{n} \mathrm{~d} t-\left(\hat{p}_{1}(\alpha), p_{1}(\alpha)\right)_{n}+\left(\hat{p}_{1}(0), p_{1}(0)\right)_{n} \\
& =\int_{0}^{\alpha}\left(\hat{p}_{1}(t), Q_{2}(t) z_{1}(t)\right)_{n} \mathrm{~d} t-\left(\hat{p}_{1}(\alpha), p_{1}(\alpha)\right)_{n}+\left(\hat{p}_{1}(0), p_{1}(0)\right)_{n}
\end{aligned}
$$

Subtracting from (0.63) equality (0.64), we obtain

$$
0=\left(\hat{p}_{1}(\alpha), p_{1}(\alpha)\right)_{n}-\left(z_{1}(\alpha), \hat{\varphi}_{1}(\alpha)\right)_{n}-\left(\hat{p}_{1}(0), p_{1}(0)\right)_{n}+\left(z_{1}(0), \hat{\varphi}_{1}(0)\right)_{n}+\int_{0}^{\alpha}\left(z_{1}(t), f^{(0)}(t)\right)_{n} \mathrm{~d} t
$$

or

$$
\int_{0}^{\alpha}\left(z_{1}(t), f^{(0)}(t)\right)_{n} \mathrm{~d} t+\left(\hat{p}_{1}(\alpha), p_{1}(\alpha)\right)_{n}-\left(z_{1}(\alpha), \hat{\varphi}_{1}(\alpha)\right)_{n}=\left(\hat{p}_{1}(0), p_{1}(0)\right)_{n}-\left(z_{1}(0), \hat{\varphi}_{1}(0)\right)_{n} .
$$

Since

$$
\begin{gathered}
\left(z_{1}(0), \hat{\varphi}_{1}(0)\right)_{n}=\left(\bar{B}_{0} z_{1}(0), B_{0} \hat{\varphi}_{1}(0)\right)_{m}=\left(\bar{B}_{0} z_{1}(0), Q_{0}^{-1} \bar{B}_{0} \hat{p}_{1}(0)\right)_{m}+\left(\bar{B}_{0} z_{1}(0), f_{0}^{(0)}\right)_{m}, \\
\left(\hat{p}_{1}(0), p_{1}(0)\right)_{n}=\left(\bar{B}_{0} \hat{p}_{1}(0), B_{0} p_{1}(0)\right)_{m}=\left(\bar{B}_{0} \hat{p}_{1}(0), Q_{0}^{-1} \bar{B}_{0} z_{1}(0)\right)_{m}
\end{gathered}
$$

we can use the latter equalities, (0.65), and the fact that $Q_{0}^{-1}$ is a symmetric matrix to obtain

$$
\left(\hat{p}_{1}(\alpha), p_{1}(\alpha)\right)_{n}-\left(z_{1}(\alpha), \hat{\varphi}_{1}(\alpha)\right)_{n}=-\int_{0}^{\alpha}\left(z_{1}(t), f^{(0)}(t)\right)_{n} \mathrm{~d} t-\left(\bar{B}_{0} z_{1}(0), f_{0}^{(0)}\right)_{m} .
$$

Performing a similar analysis, one can prove that

$$
-\left(p_{4}(\beta), \hat{p}_{4}(\beta)\right)_{n}+\left(z_{4}(\beta), \hat{\varphi}_{4}(\beta)\right)_{n}=-\int_{\beta}^{T}\left(z_{4}(t), f^{(0)}(t)\right)_{n} \mathrm{~d} t+\left(\bar{B}_{1} z_{1}(T), f_{1}^{(0)}\right)_{n-m} .
$$

From (0.66), (0.67), and (0.62) and the expression for $\hat{c}$, it follows

$$
\overline{\overline{(a, \varphi(s))_{n}}}=\left(a, \hat{\varphi}_{2}(s)\right)_{n} \text {. }
$$

The theorem is proved.

As is easily seen from (0.58), the functions $\hat{p} \in\left(H^{1}(0, T)\right)^{n}$ and $\hat{\varphi} \in\left(H^{1}(0, T)\right)^{n}$ defined by

$$
\hat{\varphi}(t):= \begin{cases}\hat{\varphi}_{1}(t), & 0 \leq t \leq \alpha ; \\ \hat{\varphi}_{2}(t), & \alpha<t \leq s ; \\ \hat{\varphi}_{3}(t), & s<t \leq \beta ; \\ \hat{\varphi}_{4}(t), & \beta<t \leq T ;\end{cases}
$$

and

$$
\hat{p}(t):= \begin{cases}\hat{p}_{1}(t), & 0 \leq t \leq \alpha ; \\ \hat{p}_{2}(t), & \alpha<t \leq s ; \\ \hat{p}_{3}(t), & s<t \leq \beta ; \\ \hat{p}_{4}(t), & \beta<t \leq T ;\end{cases}
$$

satisfy the following uniquely solvable BVP:

$$
\begin{aligned}
& L^{*} \hat{p}(t)=\chi_{(\alpha, \beta)}(t) H^{\mathrm{T}}(t) Q(t)(y(t)-H(t) \hat{\varphi}(t)), \quad 0<t<T, \\
& \hat{B}_{0} \hat{p}(0)=0, \quad \hat{B_{1}} \hat{p}(T)=0, \\
& L \hat{\varphi}(t)=Q_{2}^{-1}(t) \hat{p}(t)+f^{(0)}(t), \quad 0<t<T, \\
& B_{0} \hat{\varphi}(0)=Q_{0}^{-1} \bar{B}_{0} \hat{p}(0)+f_{0}^{(0)}, \\
& B_{1} \hat{\varphi}(T)=-Q_{1}^{-1} \bar{B}_{1} \hat{p}(T)-f_{1}^{(0)}
\end{aligned}
$$

at realizations $y$ that belong with probability 1 to space $\left(L^{2}(\alpha, \beta)\right)^{l}$. 
Thus, Theorem 4.2 can be restated in the following form.

Theorem 4.2' The minimax mean square estimate of expression $(a, \varphi(s))_{n}$ has the form

$$
\overline{\overline{(a, \varphi(s))_{n}}}=(a, \hat{\varphi}(s))_{n},
$$

where vector-function $\hat{\varphi}(t)$ is determined from the solution to problem (0.68).

Remark. Function $\hat{\varphi}(t)$ can be taken as a good, in certain sense, estimate of solution $\varphi(t)$ of initial BVP (0.1), (0.2) on $[0, T]$.

As an example, consider the case when a vector-function $y(t)=H(t) \varphi(t)+\xi(t)$ is observed on an interval $(0, T)$, where a vector-function $\varphi(t)$ with values in $\mathbb{R}^{n}$ is a solution to the BVP

$$
L_{1} \varphi=f(t), \quad \varphi(0)=f_{0}, \quad \varphi(T)=f_{1},
$$

and operator $L_{1}$ is defined by the relation

$$
L_{1} \varphi(t)=-\varphi^{\prime \prime}(t)+q(t) \varphi(t)
$$

where $q(t)$ is a positive definite $n \times n$-matrix whose entries are continuous functions on $[0, T]$.

Note that this problem has the unique classical solution if $f(t)$ is continuous on $[0, T]$ and the unique generalized solution if $f \in\left(L_{2}(0, T)\right)^{n}$.

Assume that, as well as in the previous case, $H(t)$ is an $l \times n$ matrix with the entries that are continuous functions on $[\alpha, \beta]$ and $\xi(t)$ is a random vector process with zero expectation $\mathbb{E} \xi(t)$ and unknown $l \times l$ correlation matrix $R(t, s)=\mathbb{E} \xi(t) \xi^{\mathrm{T}}(s)$. Assume also that domains $V$ and $G$ are given in the form $(0.25)$ and (0.26) where matrices $Q_{0}, Q_{1}$, and $Q_{2}(t)$ entering (0.26) have dimensions $n \times n, f_{0}^{(0)}=0, f_{1}^{(0)}=0$, and $f^{(0)}(t)=0$.

Write Equation (0.69) as a first-order system by setting $\varphi_{1}(t)=\varphi^{\prime}(t), \varphi_{2}(t)=\varphi(t)$ and introducing a vector-function

$$
\begin{gathered}
\tilde{\varphi}(t)=\left(\begin{array}{c}
\varphi_{1}(t) \\
\varphi_{2}(t)
\end{array}\right), \quad \frac{\mathrm{d} \tilde{\varphi}(t)}{\mathrm{d} t}=\left(\begin{array}{c}
\frac{\mathrm{d} \varphi_{1}(t)}{\mathrm{d} t} \\
\frac{\mathrm{d} \varphi_{2}(t)}{\mathrm{d} t}
\end{array}\right), \\
\tilde{f}(t)=\left(\begin{array}{c}
-f(t) \\
0
\end{array}\right), \quad \tilde{f}_{0}=\left(\begin{array}{c}
0 \\
f_{0}
\end{array}\right), \quad \tilde{f}_{1}=\left(\begin{array}{l}
0 \\
f_{1}
\end{array}\right)
\end{gathered}
$$

with $2 n$ components, a vector $\tilde{a}=(0, a)$ with $2 n$ components, a $2 n \times 2 n$-matrix

$$
\tilde{A}(t)=\left(\begin{array}{cc}
O_{n, n} & -q(t) \\
-E_{n} & O_{n, n}
\end{array}\right),
$$

matrices $B_{0}=B_{1}=\bar{B}_{0}=\bar{B}_{1}=\left(O_{n, n}, E_{n}\right)$, and $\hat{B}_{0}=\hat{B}_{1}=\left(E_{n}, O_{n, n}\right)$. Then system (0.69) can be written as

$$
\begin{aligned}
\tilde{L} \tilde{\varphi}(t):=\frac{\mathrm{d} \tilde{\varphi}(t)}{\mathrm{d} t}+\tilde{A} \tilde{\varphi}(t) & =\tilde{f}(t), \quad B_{0} \tilde{\varphi}(0)=\tilde{f}_{0}, \\
B_{1} \tilde{\varphi}(T) & =\tilde{f}_{1} .
\end{aligned}
$$

Applying Theorems 1 and 2 and performing necessary transformations in the resulting equations that are similar to (0.46) and (0.58) (in terms of the designations introduced above) we prove the following

Theorem 4.3 The minimax mean square estimate of expression $(a, \varphi(s))_{n}$ has the form

$$
\overline{\overline{(a, \varphi(s))_{n}}}=\int_{0}^{s}\left(\hat{u}_{1}(t), y(t)\right)_{l} \mathrm{~d} t+\int_{s}^{T}\left(\hat{u}_{2}(t), y(t)\right)_{l} \mathrm{~d} t=\left(a, \hat{\varphi}_{2}(s)\right)_{n},
$$

where

$$
\sigma^{2}=\left(a, p_{2}(s)\right)_{n},
$$


$\hat{u}_{1}(t)=Q(t) H(t) p_{2}(t), \quad \hat{u}_{2}(t)=Q(t) H(t) p_{3}(t), \quad$ and vector-functions $\quad p_{2}(t), \quad p_{3}(t)$ and $\hat{\varphi}_{2}(t)$ are determined from the solutions to the problems

$$
\begin{gathered}
L_{1} z_{2}(t)=-H^{\mathrm{T}}(t) Q(t) H(t) p_{2}(t), \quad 0<t<s, z_{2}(0)=0, \\
L_{1} z_{3}(t)=-H^{\mathrm{T}}(t) Q(t) H(t) p_{3}(t), \quad s<t<T, \\
z_{2}(s)-z_{3}(s)=a, \quad z_{3}(T)=0, \\
L_{1} p_{2}(t)=Q_{2}^{-1}(t) z_{2}(t), \quad 0<t<s, p_{2}(0)=Q_{0}^{-1} z_{2}(0), \\
L_{1} p_{3}(t)=Q_{2}^{-1}(t) z_{3}(t), \quad s<t<T, \\
p_{2}(s)=p_{3}(s), \quad p_{3}(T)=-Q_{1}^{-1} z_{3}(T),
\end{gathered}
$$

and

$$
\begin{gathered}
L_{1} \hat{p}_{2}(t)=H^{\mathrm{T}}(t) Q(t)\left(y(t)-H(t) \hat{\varphi}_{2}(t)\right), \quad 0<t<s, \\
\hat{p}_{2}(0)=0, \\
L_{1} \hat{p}_{3}(t)=H^{\mathrm{T}}(t) Q(t)\left(y(t)-H(t) \hat{\varphi}_{3}(t)\right), \quad s<t<T, \\
\hat{p}_{3}(s)=\hat{p}_{2}(s), \quad \hat{p}_{3}(T)=0, \\
L_{1} \hat{\varphi}_{2}(t)=Q_{2}^{-1}(t) \hat{p}_{2}(t), \quad 0<t<s, \quad \hat{\varphi}_{2}(0)=Q_{0}^{-1} \hat{p}_{2}(0), \\
L_{1} \hat{\varphi}_{3}(t)=Q_{2}^{-1}(t) \hat{p}_{3}(t), \quad s<t<T, \\
\hat{\varphi}_{3}(s)=\hat{\varphi}_{2}(s), \quad \hat{\varphi}_{3}(T)=-Q_{1}^{-1} \hat{p}_{3}(T),
\end{gathered}
$$

respectively.

\section{Minimax Mean Square Estimates of Solutions Subject to Incomplete Restrictions on Unknown Parameters}

Assume again that observations have form (0.24) and unknown parameters $f_{0}, f_{1}$, and $f(t)$ belong to the domain

$$
G=\left\{\tilde{F}=\left(\tilde{f}_{0}, \tilde{f}_{1}, \tilde{f}\right): \int_{0}^{T}\left(Q_{2}(t) \tilde{f}(t), \tilde{f}(t)\right) \mathrm{d} t \leq 1\right\},
$$

where $Q_{2}(t)$ is given in (0.26). The correlation function of process $\xi(t)$ belongs to domain $(0.25)$. Introduce the set

$$
U=\left\{u(\cdot): \bar{B}_{0} z_{1}(0, u)=0, \bar{B}_{1} z_{4}(T, u)=0\right\}
$$

here $u(t)=\left\{\begin{array}{ll}u_{1}(t), & \alpha<t<s, \\ u_{2}(t), & s<t<\beta,\end{array}\right.$ where $z_{i}(t, u), i=\overline{1,4}, \quad$ is the solution to BVP (0.29).

\section{Lemma 5.1}

$$
\sigma(u, c)= \begin{cases}\infty, & u \notin U, \\ \sigma_{1}(u, c), & u \in U,\end{cases}
$$

where

$$
\sigma_{1}(u, c)=\int_{0}^{T}\left(Q_{2}^{-1} \tilde{z}(t, u), \tilde{z}(t, u)\right) \mathrm{d} t+\int_{\alpha}^{\beta}\left(Q^{-1}(t) u(t), u(t)\right) \mathrm{d} t+c^{2}=J(u)+c^{2} .
$$

This lemma can be proved using formula (0.39).

Lemma 5.2 $U$ is a convex closed set in the space $\left(L_{2}(\alpha, \beta)\right)^{l}$.

Proof. The convexity of set $U$ is obvious. Let us prove that this set is closed.

Note that functions $z_{1}(0, u)$ and $z_{4}(T, u)$ can be represented as 


$$
\begin{aligned}
& z_{1}(0, u)=a_{1}+\int_{\alpha}^{\beta} \Phi_{1}(t) u(t) \mathrm{d} t, \\
& z_{4}(T, u)=a_{2}+\int_{\alpha}^{\beta} \Phi_{2}(t) u(t) \mathrm{d} t,
\end{aligned}
$$

where $\Phi_{1}(t)$ and $\Phi_{2}(t)$ are known matrix functions with the elements from $L_{2}(\alpha, \beta)$ and $a_{1}$ and $a_{2}$ are vectors. Expression (0.75) can be obtained if we introduce a vector $z_{0}$ such that $z_{1}(0, u)=z_{0}$. Then $z_{1}(\alpha, u)=\Phi(\alpha, 0) z_{0}$,

$$
\begin{aligned}
z_{2}(s, u) & =\Phi(s, \alpha) z_{2}(\alpha, u) \\
& =\Phi(s, \alpha) z_{1}(\alpha, u)+\int_{\alpha}^{s} \Phi(s, \tau) H^{\mathrm{T}}(\tau) u_{1}(\tau) \mathrm{d} \tau \\
& =\Phi(s, 0) z_{0}+\int_{\alpha}^{s} \Phi(s, \tau) H^{\mathrm{T}}(\tau) u_{1}(\tau) \mathrm{d} \tau,
\end{aligned}
$$

where $\Phi(t, \tau)$ is a solution to the equation

$$
\frac{\mathrm{d} \Phi(t, \tau)}{\mathrm{d} t}=-A^{*}(t) \Phi(t, \tau), \quad \Phi(\tau, \tau)=E
$$

and $E$ is the unit matrix. Next,

$$
z_{4}(T, u)=\Phi(T, 0) z_{0}+\int_{\alpha}^{\beta} \Phi(T, \tau) H^{\mathrm{T}}(\tau) u(\tau) \mathrm{d} \tau+\Phi(T, s) a .
$$

Since BVP (0.29) is uniquely solvable, there exists one and only one vector $z_{0}$ satisfying the system of algebraic equations

$$
\left\{\begin{array}{l}
\bar{B}_{0} z_{0}=0, \\
B_{1} \Phi(T, 0) z_{0}=-\int_{\alpha}^{\beta} B_{1} \Phi(t, \tau) H^{\mathrm{T}}(\tau) u(\tau) \mathrm{d} \tau-B_{1} \Phi(T, s) a .
\end{array}\right.
$$

Solving this system we determine $z_{0}$ in the form

$$
z_{0}=b+\int_{\alpha}^{\beta} \Phi_{0}(\tau) u(\tau) \mathrm{d} \tau,
$$

where $\Phi_{0}(\tau)$ is a known matrix function continuous on $[\alpha, \beta]$ and $b$ is a known vector. Taking into account this equality, we obtain expressions (0.75). From these relationships, it follows that if a sequence $u_{n}(t)$ converges in $L_{2}(\alpha, \beta)$ to a function $u_{0}(t)$, then

$$
\begin{aligned}
& \lim _{n \rightarrow \infty} \bar{B}_{0} z_{1}\left(0, u_{n}\right)=\bar{B}_{0} z_{1}\left(0, u_{0}\right), \\
& \lim _{n \rightarrow \infty} \bar{B}_{1} z_{4}\left(0, u_{n}\right)=\bar{B}_{1} z_{4}\left(0, u_{0}\right),
\end{aligned}
$$

which proves that $U$ is a closed set.

Assume now that $U$ is nonempty. Then the following statement is valid.

Theorem 5.1 There exists the unique minimax mean square estimate of expression $(a, \varphi(s))$ which can be represented in the form (0.44) at $\hat{c}=0$, where vector-functions $p_{2}(t)$ and $p_{3}(t)$ solve the equations

$$
\begin{aligned}
& L^{*} z_{1}(t)=0, \quad 0<t<\alpha, \quad \hat{B}_{0} z_{1}(0)=0, \\
& L^{*} z_{2}(t)=-H^{\mathrm{T}}(t) Q(t) H(t) p_{2}(t), \quad \alpha<t<s, \quad z_{2}(\alpha)=z_{1}(\alpha), \\
& L^{*} z_{3}(t)=-H^{\mathrm{T}}(t) Q(t) H(t) p_{3}(t), \quad s<t<\beta, \quad z_{3}(s)=z_{2}(s)-a, \\
& L^{*} z_{4}(t)=0, \quad \beta<t<T, \quad z_{4}(\beta)=z_{3}(\beta), \\
& \hat{B}_{1} z_{4}(T)=0, \quad \bar{B}_{0} z_{1}(0), \quad \bar{B}_{1} z_{4}(T)=0, \\
& L p_{1}(t)=Q_{2}^{-1}(t) z_{1}(t), \quad 0<t<\alpha, \\
& L p_{2}(t)=Q_{2}^{-1} t z_{2}(t), \quad \alpha<t<s, \quad p_{2}(\alpha)=p_{1}(\alpha), \\
& L p_{3}(t)=Q_{2}^{-1}(t) z_{3}(t), \quad s<t<\beta, \quad p_{3}(s)=p_{2}(s), \\
& L p_{4}(t)=Q_{2}^{-1}(t) z_{4}(t), \quad s<t<\beta .
\end{aligned}
$$


Proof. Similarly to Theorem 4.1 one can show that for $u \in U$ the following equality holds

$$
\sigma(u, c)=J(u)+c^{2},
$$

where

$$
\begin{aligned}
J(u)= & \int_{0}^{\alpha}\left(Q_{2}^{-1}(t) z_{1}(t, u), z_{1}(t, u)\right)_{n} \mathrm{~d} t+\int_{\alpha}^{s}\left(Q_{2}^{-1}(t) z_{2}(t, u), z_{2}(t, u)\right)_{n} \mathrm{~d} t \\
& +\int_{s}^{\beta}\left(Q_{2}^{-1}(t) z_{3}(t, u), z_{3}(t, u)\right)_{n} \mathrm{~d} t+\int_{\beta}^{T}\left(Q_{2}^{-1}(t) z_{4}(t, u), z_{4}(t, u)\right)_{n} \mathrm{~d} t \\
& +\int_{\alpha}^{s}\left(Q^{-1}(t) u_{1}(t), u_{1}(t)\right) \mathrm{d} t+\int_{s}^{\beta}\left(Q^{-1}(t) u_{2}(t), u_{2}(t)\right) \mathrm{d} t,
\end{aligned}
$$

and $z_{i}(t, u), i=\overline{1,4}$, are solutions to Equations $(0.29)$ at $\bar{B}_{0} z_{1}(0, u)=0$ and $\bar{B}_{1} z_{4}(T, u)=0$. $J(u)$ is a strictly convex lower semicontinuous functional on a closed convex set $U$ and $\lim _{\|u\| \rightarrow \infty} J(u)=\infty$. Therefore there exists one and only one vector $\hat{u}$ such that $\min _{u \in U} J(u)=J(\hat{u})$. This vector can be determined from the relationship

$$
\left.\frac{\mathrm{d}}{\mathrm{d} \tau} J_{\mu}\left(\hat{u}_{1}+\tau v_{1}, \hat{u}_{2}+\tau v_{2}\right)\right|_{\tau=0} \equiv 0, \quad \forall v=\left(v_{1}, v_{2}\right) \in H,
$$

where

$$
J_{\mu}(u)=J\left(u_{1}, u_{2}\right)+\left(\mu_{1}, \bar{B}_{0} z_{1}(0, u)\right)+\left(\mu_{2}, \bar{B}_{1} z_{4}(T, u)\right),
$$

$\mu=\left(\mu_{1}, \mu_{2}\right), \quad \mu_{1} \in \mathbb{R}^{m}$, and $\mu_{2} \in \mathbb{R}^{n-m}$ are Lagrange multipliers.

Further analysis is similar to the proof of Theorem 4.1. Let vector-functions $\hat{p}_{i}(t)$ and $\hat{\varphi}_{i}(t), i=\overline{1,4}$, be solutions to the system

$$
\begin{aligned}
& L^{*} \hat{p}_{1}(t)=0, \quad 0<t<\alpha, \\
& L^{*} \hat{p}_{2}(t)=H^{\mathrm{T}}(t) Q(t)\left[y(t)-H(t) \hat{\varphi}_{2}(t)\right], \quad \alpha<t<s, \\
& L^{*} \hat{p}_{3}(t)=H^{\mathrm{T}}(t) Q(t)\left[y(t)-H(t) \hat{\varphi}_{3}(t)\right], \quad s<t<\beta, \\
& L^{*} \hat{p}_{4}(t)=0, \quad \beta<t<T, \\
& L \hat{\varphi}_{1}(t)=Q_{2}^{-1}(t) \hat{p}_{1}(t), \quad 0<t<\alpha, \\
& L \hat{\varphi}_{2}(t)=Q_{2}^{-1}(t) \hat{p}_{2}(t), \quad \alpha<t<s, \\
& L \hat{\varphi}_{3}(t)=Q_{2}^{-1}(t) \hat{p}_{3}(t), \quad s<t<\beta, \\
& L \hat{\varphi}_{4}(t)=Q_{2}^{-1}(t) \hat{p}_{4}(t), \quad \beta<t<T, \\
& \hat{B}_{0} \hat{p}_{1}(0)=0, \quad \hat{p}_{2}(\alpha)=\hat{p}_{1}(\alpha), \\
& \hat{p}_{3}(s)=\hat{p}_{2}(s), \quad \hat{p}_{4}(\beta)=\hat{p}_{3}(\beta), \\
& \hat{B}_{1} \hat{p}_{1}(T)=0, \quad \bar{B}_{0} \hat{p}_{1}(0)=0, \quad \bar{B}_{1} \hat{p}_{4}(T)=0, \\
& \hat{\varphi}_{2}(\alpha)=\hat{\varphi}_{1}(\alpha), \quad \hat{\varphi}_{3}(s)=\hat{\varphi}_{2}(s), \quad \hat{\varphi}_{4}(\beta)=\hat{\varphi}_{3}(\beta) .
\end{aligned}
$$

Theorem 5.2 Assume that for any vector $a \in \mathbb{R}^{n}$ set $U$ is nonempty. Then system (0.77) is uniquely solvable and the equality

$$
\overline{\overline{(a, \varphi(s))}}=\left(a, \hat{\varphi}_{2}(s)\right)
$$

holds

Proof. Introduce functions $\hat{p}_{i}(t, v), i=\overline{1,4}$, as a solution to the BVP

$$
\begin{gathered}
L^{*} \hat{p}_{1}(t, v)=0, \quad 0<t<\alpha, \\
L^{*} \hat{p}_{2}(t, v)=d(t)-H^{\mathrm{T}}(t) v_{1}(t), \quad \alpha<t<s, \\
L^{*} \hat{p}_{3}(t, v)=d(t)-H^{\mathrm{T}}(t) v_{2}(t), \quad s<t<\beta,
\end{gathered}
$$




$$
\begin{gathered}
L^{*} \hat{p}_{4}(t, v)=0, \quad \beta<t<T, \\
\hat{B}_{0} \hat{p}_{1}(0, v)=0, \quad \hat{B}_{1} \bar{p}_{4}(T, v)=0, \\
\hat{p}_{2}(\alpha, v)=\hat{p}_{1}(\alpha, v), \quad \hat{p}_{3}(s, v)=\hat{p}_{2}(s, v), \\
\hat{p}_{4}(\beta, v)=\hat{p}_{3}(\beta, v),
\end{gathered}
$$

where $d(t)=H^{\mathrm{T}}(t) Q(t) y(t)$. Define a set

$$
U_{1}=\left\{v: \bar{B}_{0} \hat{p}_{1}(0, v)=0, \bar{B}_{1} \hat{p}_{4}(T, v)=0\right\} .
$$

Since $U$ is nonempty, the same is valid for $U_{1}$ for any vector $a$. Similarly to the case of $U$ one can show that $U_{1}$ is a convex closed set. Denote by $J_{1}(v)$ the functional of the form

$$
\begin{aligned}
J_{1}(v)= & \int_{0}^{\alpha}\left(Q_{2}^{-1}(t) \hat{p}_{1}(t, v), \hat{p}_{1}(t, v)\right) \mathrm{d} t+\int_{\alpha}^{s}\left(Q_{2}^{-1}(t) \hat{p}_{2}(t, v), \hat{p}_{2}(t, v)\right) \mathrm{d} t \\
& +\int_{s}^{\beta}\left(Q_{2}^{-1}(t) \hat{p}_{3}(t, v), \hat{p}_{3}(t, v)\right) \mathrm{d} t+\int_{\beta}^{T}\left(Q_{2}^{-1}(t) \hat{p}_{4}(t, v), \hat{p}_{4}(t, v)\right) \mathrm{d} t \\
& +\int_{\alpha}^{s}\left(Q^{-1}(t) v_{1}(t), v_{1}(t)\right) \mathrm{d} t+\int_{s}^{\beta}\left(Q^{-1}(t) v_{2}(t), v_{2}(t)\right) \mathrm{d} t .
\end{aligned}
$$

One can show, following Theorem 5.1, that on set $U_{1}$ there is one and only one point of minimum of functional $J_{1}(v)$, namely,

$$
\hat{v}_{1}(t)=Q^{-1}(t) H(t) \hat{\varphi}_{2}(t), \quad \hat{v}_{2}(t)=Q^{-1}(t) H(t) \hat{\varphi}_{3}(t),
$$

where functions $\hat{\varphi}_{2}(t)$ and $\hat{\varphi}_{3}(t)$ are determined from system (0.77). The proof of the equality

$$
\overline{\overline{(a, \varphi(s))_{n}}}=\left(a, \hat{\varphi}_{2}(s)\right)_{n}
$$

is similar to that in Theorem 4.2.

\section{Conclusions}

For a system described by a one-dimensional two-point BVP with decoupling boundary conditions at the endpoints of the interval and quadratic restrictions imposed on the unknown deterministic data and the second moments of observation noise, we have obtained guaranteed mean square estimates of inner product $(a, \varphi(s))$, where $\varphi(s)$ is the unknown solution of the BVP at a point $s \in(0, T)$ and $a \in \mathbb{R}^{n}$. Guaranteed estimates are obtained using the duality of the problems of estimation and optimal control. We have shown that guaranteed mean square estimates and estimation errors are expressed via solutions to special optimal control problems for conjugate BVPs. The solutions to these optimal control problems enable one to find explicit expressions for estimates and estimation errors both for distributed and point observations.

The obtained results are applied to minimax estimation of solutions of two-point BVPs for linear ordinary second-order differential equations.

Methods and results of the paper may be used for estimation under uncertainties of the states of the systems described by more general linear BVPs for different classes of functional--differential equations; in particular, for systems of differential equations with impulse perturbations, differential equations with multipoint conditions, and in several other cases.

\section{Results of Numerical Experiments}

Let realizations of the random variables

$$
y_{k}=\int_{0}^{1} g_{k}(x) \varphi(x) \mathrm{d} x+\xi_{k}, \quad k=1, \cdots, N,
$$

be observed. Here $\xi_{k}$ are independent random variables for which $\mathbb{E} \xi_{k}=0, \mathbb{E} \xi_{k}^{2}=r_{k}^{2} ; \varphi(x)$ is a solution of the BVP

$$
-\varphi^{\prime \prime}(x)+\omega^{2} \varphi(x)=f(x)
$$




$$
\varphi(0)=\varphi(1)=0
$$

and

$$
g_{k}(x)=\sqrt{2} \sin k \pi x, \quad k=1,2, \cdots
$$

are eigenfunctions of the operator $-\frac{\mathrm{d}^{2}}{\mathrm{~d} x^{2}}: H_{0}^{2}(0,1) \rightarrow L^{2}(0,1)$, where $H_{0}^{2}(0,1)=\left\{u \in H^{2}(0,1): u(0)=u(1)=0\right\}$.

We assume that function $f(x)$ is not known exactly and is chosen arbitrarily from the set $G=\left\{f(x): \int_{0}^{1} f^{2}(x) \mathrm{d} x \leq q^{2}\right\}$, where $q$ is a certain constant.

Applying the technique similar to the estimation methods developed in Section 4, it is possible to obtain expressions for the minimax mean square estimates in the case when observations have the form $(0.78)$. In particular, in this case the function $\hat{\varphi}$ which approximates the solution $\varphi$ of BVP (0.79)-(0.80) on the interval $[0,1]$ is determined from the system

$$
\begin{gathered}
-\hat{p}^{\prime \prime}(x)+\omega^{2} \hat{p}(x)=\sum_{k=1}^{N} r_{k}^{-2} g_{k}(x)\left(y_{k}-\left(g_{k}, \hat{\varphi}\right)_{L^{2}(0,1)}\right), \\
\hat{p}(0)=\hat{p}(1)=0, \\
-\hat{\varphi}^{\prime \prime}(x)+\omega^{2} \hat{\varphi}(x)=q^{2} \hat{p}(x), \\
\hat{\varphi}(0)=\hat{\varphi}(1)=0,
\end{gathered}
$$

and can be represented in the form

$$
\hat{\varphi}(x)=\sum_{k=1}^{N}\left(\frac{r_{k}^{2}\left((k \pi)^{2}+\omega^{2}\right)^{2}}{q^{2}}+1\right)^{-1} g_{k}(x) y_{k} .
$$

The exact solution $\varphi$ and its estimate $\hat{\varphi}$ (bold curves) calculated on the basis of the modelled observations are presented in Figure 1 and Figure 2. Calculations are performed at $q=1, w=1$, and $N=5$ for $k=1, \cdots, 5$

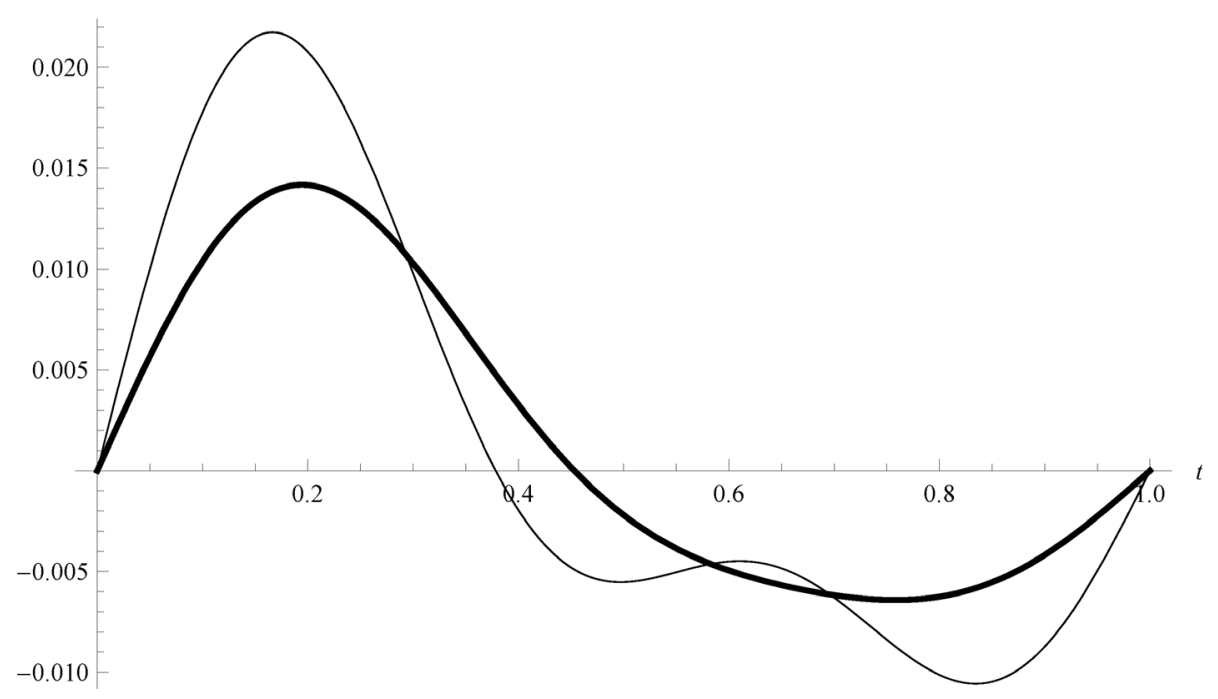

Figure 1. Exact solution $\varphi$ and its estimate $\hat{\varphi}$ (bold curve) calculated at $q=1, w=1, N=5$, $r_{k}=r=\frac{1}{100}(k=1, \ldots, 5)$, and $f(x)=\frac{1}{2000} \sin \pi x+\frac{1}{2} \sin 2 \pi x+\frac{1}{2} \sin 3 \pi x+\sin 4 \pi x$. 


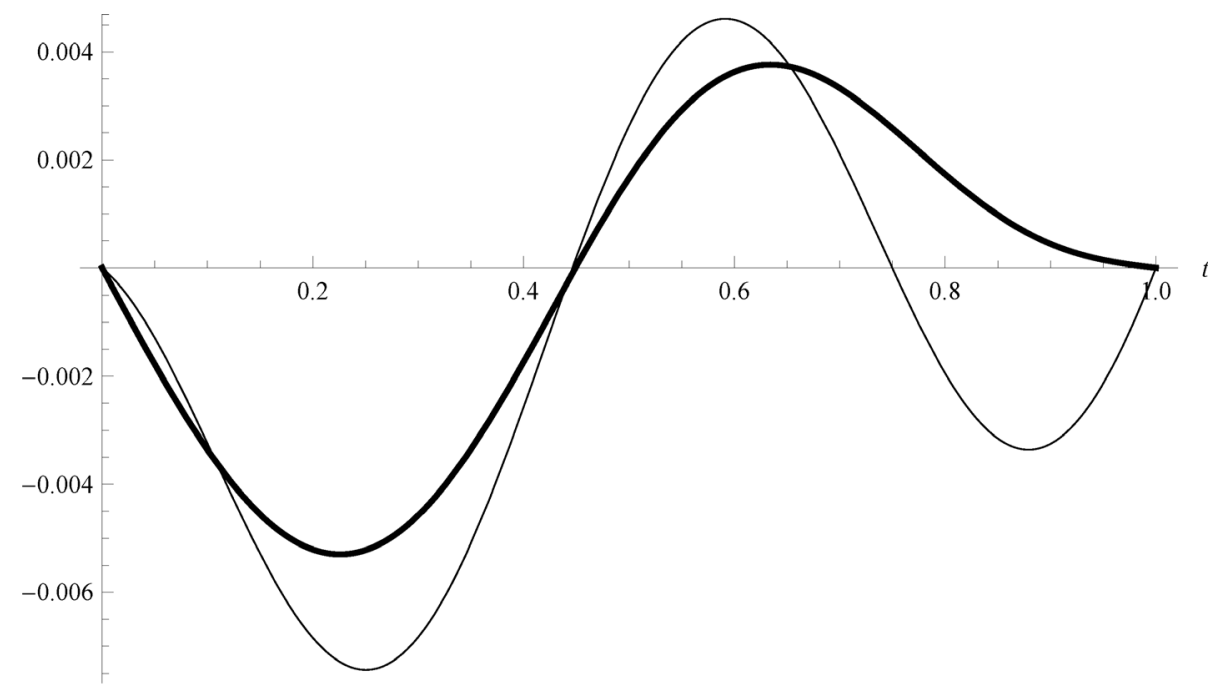

Figure 2. Exact solution $\varphi$ and its estimate $\hat{\varphi}$ (bold curve) calculated at $q=1, w=1, N=5$,

$r_{k}=r=\frac{1}{500}(k=1, \ldots, 5)$, and $f(x)=\frac{1}{2} \cos \left(10 x+\frac{1}{2}\right)$.

using the parameters $r_{k}=r=\frac{1}{100}$ and $f(x)=\frac{1}{2000} \sin \pi x+\frac{1}{2} \sin 2 \pi x+\frac{1}{2} \sin 3 \pi x+\sin 4 \pi x \quad$ (Figure 1) and $r_{k}=r=\frac{1}{500}$ and $f(x)=\frac{1}{2} \cos \left(10 x+\frac{1}{2}\right)$ (Figure 2).

As can be seen from these figures, parameter $r$ plays the crucial role as far as the estimation quality is concerned. In fact, this parameter directly influences the signal-to-noise ratio.

The calculations were performed using Wolfram Mathematica.

\section{References}

[1] Kalman, R. (1960) A New Approach to Liner Filtering and Prediction Problems. Journal of Basic Engineering, 82, 35-45.

[2] Kalman, R. and Bucy, R. (1961) New Results in Liner Filtering and Prediction Theory. Transactions of the ASME-Journal of Basic Engineering, 83, 95-108. http://dx.doi.org/10.1115/1.3658902

[3] Krasovskii, N. (1968) Theory of Motion Control. Nauka, Moscow.

[4] Kurzhanskii, A. (1977) Control and Observation under Uncertainties. Nauka, Moscow.

[5] Kirichenko, N. and Nakonechnyi, O. (1977) A Minimax Approach to Recurrent Estimation of the States of Linear Dynamical Systems. Kibernetika, 4, 52-55.

[6] Nakonechnyi, O. (1979) Minimax Estimates in Systems with Distributed Parameters. Preprint 79, Acad. Sci. USSR, Inst. Cybernetics, Kyiv, 55 p.

[7] Kuntsevich, V. (2005) Accuracy of Construction of Approximating Models under Bounded Measurement Noises. Automation and Remote Control, 66, 791-798. http://dx.doi.org/10.1007/s10513-005-0123-0

[8] Kurzhanski, A. and Valyi, I. (1997) Ellipsoidal Calculus for Estimation and Control. Birkhauser Verlag, Basel. http://dx.doi.org/10.1007/978-1-4612-0277-6

[9] Nakonechnyi, O., Podlipenko, Y. and Shestopalov, Y. (2009) Estimation of Parameters of Boundary Value Problems for Linear Ordinary Differential Equations with Uncertain Data. arXiv:0912.2872v1, 1-72.

[10] Podlipenko, Y. (2005) Minimax Estimation of Right-Hand Sides of Noetherian Equations in a Hilbert Space under Uncertainty Conditions. Reports of the National Academy of Sciences of Ukraine, 12, 36-44.

[11] Basar, T. and Bernhard, P. (1991) H-Optimal Control and Related Minimax Design Problems. Birkhäuser, Basel.

[12] Fedoryuk, M. (1985) Ordinary Differential Equations. Nauka, Moscow.

[13] Naimark, M. (1969) Linear Differential Operators. Nauka, Moscow. 
[14] Krein, S. (1971) Linear Equations in the Banach Space. Nauka, Moscow.

[15] Atkinson, F. (1964) Discrete and Coninuous Boundary Value Problems. Academic Press, New York.

[16] Hutson, V., Pym, J. and Cloud, M. (2005) Applications of Functional Analysis and Operator Theory. Vol. 200, 2nd Edition, Mathematics in Science and Engineering, Elsevier Science, Amsterdam.

[17] Lions, J. (1968) Controle optimal de systémes gouvernés par des équations aux dérivées partielles. Dunod, Paris. 